\title{
Effectiveness of diet and physical activity interventions among Chinese-origin populations living in high income countries: a systematic review
}

\author{
Jeannette M. Beasley ${ }^{1 *}\left(\mathbb{D}\right.$, Janelle M. Wagnild ${ }^{2}$, Tessa M. Pollard², Timothy R. Roberts ${ }^{3}$ and Nasima Ahkter ${ }^{2}$
}

\begin{abstract}
Background: This review examines the effectiveness of diet and physical activity interventions to reduce cardiometabolic risk among Chinese immigrants and their descendants living in high income countries. The objective of this review is to provide information to help build future interventions aimed at improving diet and increasing physical activity levels among Chinese immigrants.

Methods: Outcomes included BMI, weight, waist circumference (WC), waist-hip ratio (WHR), cholesterol (LDL, HDL), systolic and diastolic blood pressure (SBP, DBP), hemoglobin A1c (HgbA1c), fasting blood glucose (FBG), and HOMA-IR. Six databases were systematically searched from database inception to date of search (February 2020). Meta-analyses used random effect models to estimate pooled effects of outcomes with 95\% confidence intervals. The outcomes assessed were changes in mean outcomes (post-intervention versus baseline) among the intervention group versus control groups.

Results: Twenty-one articles were included for synthesis, and eight of these were included in the meta-analysis. Among children/adolescents, there were no significant effects of intervention for any of the outcomes having sufficient data for meta-analysis (BMI, WHR, SBP, and DBP). Among adults, the pooled effect including three studies showed significant changes in BMI (effect size $\left.=-1.14 \mathrm{~kg} / \mathrm{m}^{2} ;(95 \% \mathrm{Cl}:-2.06,-0.21), \mathrm{I}^{2}=31 \%\right)$. There were also significant effects of intervention among adults in terms of changes in SBP and DBP, as the pooled effect across three studies was $-6.08 \mathrm{mmHg}(95 \% \mathrm{Cl}-9.42,-2.73), \mathrm{I}^{2}=0 \%$ and $-3.81 \mathrm{mmHg}(95 \% \mathrm{Cl}:-6.34,-1.28), \mathrm{I}^{2}=0 \%$, respectively. Among adults there were no other significant effects among the meta-analyses conducted (weight, WC, LDL, HgbA1C, and FBG).

(Continued on next page)
\end{abstract}

\footnotetext{
* Correspondence: jeannette.beasley@nyumc.org

'Department of Medicine, NYU Grossman School of Medicine, 462 First Avenue CD 673, New York, NY 10016, USA

Full list of author information is available at the end of the article
}

(c) The Author(s). 2020 Open Access This article is licensed under a Creative Commons Attribution 4.0 International License, which permits use, sharing, adaptation, distribution and reproduction in any medium or format, as long as you give appropriate credit to the original author(s) and the source, provide a link to the Creative Commons licence, and indicate if changes were made. The images or other third party material in this article are included in the article's Creative Commons licence, unless indicated otherwise in a credit line to the material. If material is not included in the article's Creative Commons licence and your intended use is not permitted by statutory regulation or exceeds the permitted use, you will need to obtain permission directly from the copyright holder. To view a copy of this licence, visit http://creativecommons.org/licenses/by/4.0/. The Creative Commons Public Domain Dedication waiver (http://creativecommons.org/publicdomain/zero/1.0/) applies to the data made available in this article, unless otherwise stated in a credit line to the data. 
(Continued from previous page)

Conclusions: This review is the first to summarize the effectiveness of diet and physical activity interventions specifically designed for Chinese immigrants living in high income countries. There were clinically meaningful changes in BMI and blood pressure among adults, but evidence was weak for other cardiometabolic outcomes (weight, WC, LDL, HgbA1C, and FBG), and among children, there was no evidence of effect for any cardiometabolic outcomes. Given our mixed findings, more work is needed to support the design of successful interventions, particularly those targeting children and their families.

Trial registration: The systematic review protocol was registered in PROSPERO on December 17, 2018, the international prospective register of systematic reviews (registration number: CRD42018117842).

Keywords: Migrants, nutrition, Food, Exercise, Tai chi, Strength, Body mass index, Blood pressure, Lipids

\section{Background}

People of Chinese origin make up one of the fastest expanding groups in high-income countries such as the United States, Australia and Canada [1]. The cardiometabolic disease profile for this group is generally positive [1], but there are concerns about a high prevalence of type 2 diabetes identified in some studies $[2,3]$ and about increasing adiposity. While measures of adiposity such as BMI and waist circumference are generally low in Chinese-origin populations in high-income countries in comparison with other ethnic groups [3, 4], there is evidence that it increases with time living in a highincome country [4], that it is higher in those born to Chinese-origin parents in the United States than in migrants from China [4-6], and some evidence that it has been increasing faster amongst Chinese ethnic groups than amongst others [7]. People of Asian origin have a higher risk of cardiovascular disease at a given BMI relative to other ethnic subgroups [3], suggesting that strategies to improve diet and physical activity behaviors may be particularly important for those of Asian origin, including Chinese immigrants and their descendants [8].

There is good evidence of differences in physical activity and dietary practices between Chinese migrant groups and the rest of the population in a number of countries with the largest Chinese-origin populations. There was a higher prevalence of inactivity among Chinese Australians than non-Chinese Australians [3], Canadians of South-East Asian origin (including people with Chinese origins) were more likely to be physically inactive than the White population of Canada [9], those of Chinese origin reported lower levels of physical activity compared with the general population in the UK [10], and not only were NonHispanic Asians in New York City less likely to meet physical activity guidelines than non-Hispanic Whites or Blacks, but Chinese Americans were less likely to meet physical activity guidelines than other Asian subgroups [11]. Similarly, in New Zealand those of Chinese ethnicity were less likely to achieve physical activity recommendations [8].
Dietary differences are harder to characterize. Those of Chinese origin ate greater amounts of fruit and vegetables than the general population in the UK and fat intake was relatively low [12], while studies in the United States and in New Zealand found that those of Chinese ethnicity were less likely to meet recommendations for consumption of vegetables than the general population $[13,14]$. Dietary patterns change with length of residence amongst migrants from China, with migrants to Canada and the United States showing negative changes such as reduced consumption of fruit and vegetables, increased portion sizes and greater consumption of convenience foods $[14,15]$ and a survey of Chinese immigrant mothers living in NYC reported several changes in diet after immigration including a decrease in family meals [7]. Thus interventions to promote physical activity and healthful diets could be particularly beneficial for those of Chinese-origin.

Considerations for developing interventions for Chinese migrants and/or their descendants include: 1) language (whether the intervention was offered in Cantonese, Mandarin, English, etc.); 2) health literacy; 3) traditional Chinese diet; 4) migration and acculturation; and 5) traditional Chinese medicine [16]. Successful interventions may encourage maintenance of healthful dietary practices, incorporate traditional and cultural beliefs, and provide information that would enable the participants to make healthful dietary modifications [17]. Adaptations at a surface level may involve the use of vernacular phrases, role models that represent the targeted group, identifying suitable media channels and settings for recruitment, and employing ethnically matched staff to administer the program [18]. At the deep structure level, adaptations may address the opposing cultural dimensions of collectivism and individualism [18].

In the context of some concerns about diet and physical activity in those of Chinese origin living in highincome countries, and evidence that this group may benefit from tailored interventions, this review examines the effectiveness of interventions designed to modify dietary and physical activity behaviors to reduce 
cardiometabolic risk in this group. The objective of this review is to provide information to build future interventions aimed at improving the diet and increasing physical activity levels among Chinese immigrants.

\section{Methods}

The review was conducted following the PRISMA Protocol for Systematic Reviews (PRISMA) [19] and the protocol was registered in PROSPERO, International prospective register of systematic reviews (CRD4201811 7842).

\section{Information sources and search strategy}

In February 2020, co-author (TR), an experienced Medical Librarian, searched PubMed Central, Ovid Medline, Ovid Embase, CABI, Food Science Technology Cinahl and the Cochrane Central Register of Controlled Trials. The Ovid Medline Search is included as supplementary material (Supplementary Table 1) to this article. The search was not limited by language or publication date. Additionally, the citations of included articles were checked and, if relevant, were included in the review.

\section{Eligibility criteria}

This review examined diet and physical activity interventions to reduce cardiometabolic risk among Chinese immigrants living in high income countries outside of China. To this end, studies were included in the review if 1 ) they quantitatively described the effect of an intervention designed to modify dietary and/or physical activity behaviors on cardiometabolic risk factors (BMI, weight, waist circumference (WC), waist-hip ratio (WHR), LDL and/or HDL cholesterol, systolic and diastolic blood pressure (SBP and DBP), hemoglobin A1c (HgbA1c), fasting blood glucose (FBG), and HOMA-IR), and 2) the recipients of the intervention were of Chinese origin and living in a high-income economy, as defined by the World Bank [20]. Exclusion criteria were: studies involving institutionalized populations (as individuallevel control over diet and physical activity behaviors may be restricted), and studies whose samples included residents of Hong Kong, Taiwan, and Macau (as these high-income economies are special administrative regions within China). Interventions could be at any level (individual, community, policy). The only types of studies to be excluded were observational studies in which no intervention was tested. Systematic reviews and meta-analyses on related topics were tagged for review of individual studies, but the review paper itself was not included to avoid double counting of studies. Control groups were comprised of alternative combinations of diet and physical activity interventions, attention control, cross-over designs, or before/after studies.

\section{Study selection and data extraction}

Titles and abstracts were screened by four independent reviewers (JB, JW, TP, NA), with each citation receiving two votes. The full-texts of studies with relevant abstracts were assessed for eligibility by two screeners independently (JB, JW). Any conflicts were discussed and resolved through consensus of all four reviewers.

Data from studies eligible for inclusion were extracted using a data extraction form adapted from published sources such as the Cochrane review [21, 22]. If pre- and post-intervention means were not provided in the manuscript, the corresponding author was contacted to request the data. Quality assessment was determined using the Cochrane Review's Risk of Bias tool [21], and guidelines provided in the Cochrane handbook for systematic reviews of interventions were used to assess risk of bias [23]. Two reviewers (JB, JW) independently extracted outcomes by reading the full articles, tables, figures and interpretations for the findings and assessed the quality of papers to ensure consistency and to minimize individual bias. Discrepancies were resolved by consensus (TP, NA, JB, JW).

\section{Synthesis of results}

A narrative synthesis was used as it allows the compilation of data despite potential differences in research questions, design, or context in order to find a common underlying pattern. If at least two studies included the same outcome variable and pre- and post-intervention values were reported for both the intervention and control group, a meta-analysis was conducted. In cases where multiple post-intervention measurements were available, we extracted the measure that corresponded most closely to the endpoint of the intervention. We stratified analyses by age group (children/adolescents and adults).

\section{Statistical analysis}

Where meta-analysis was possible (e.g. pre-post measures were available for intervention and control groups), the analyses involved two steps. The first step was to assess mean differences (MD) in outcomes for both the intervention and control group by comparing changes in the mean as the difference between postintervention and baseline measures. For calculating MD, available adjusted or unadjusted means as reported in the included studies were used. The corresponding changes in standard deviation (SD) were not directly reported in most studies, and therefore was estimated using the formula suggested by the Cochrane handbook for systematic reviews of interventions [23]. A correlation of 0.6 between pre- and post-intervention values was assumed. The second step involved estimating the pooled effect for outcomes, where at least two 
randomized, controlled trials (RCTs) reported on the same outcome variables. The pooled effects as gain in the intervention group against the change in control group was reported as the pooled effect estimate with 95\% CIs. The study weights were equal to the inverse of the variance of effect estimate of each study as suggested by DerSimonian and Laird [24, 25]. The overall effect was interpreted as statistically significant if the $95 \%$ CIs did not include the null value of 0 (no difference) in their range. Sensitivity analyses were performed to assess whether correlation of 0.5 or 0.8 affected the interpretation of the pooled effect. Heterogeneity, i.e. variation in the intervention effects observed in the included studies, was quantified using the $\mathrm{I}^{2}$ statistic. Results are to be interpreted with caution where there is significant heterogeneity $\left(\mathrm{I}^{2}>50 \%\right)$. Meta-analyses were performed in $\mathrm{R}$ software using the 'meta' package.

\section{Results}

\section{Study selection}

After duplicates were removed, 4443 articles were identified (Fig. 1). The initial screening of titles and abstracts removed 4335 articles, leaving 107 full text articles to be screened by two reviewers independently (JB, JW). Of the full text articles reviewed, 86 articles were excluded for the reasons listed in Fig. 1. Twenty-one articles were included for synthesis, including one study reporting outcomes for both children and adults [26]. Of these, eight provided the pre- and post-intervention means for intervention and control groups, allowing for inclusion in the meta-analysis [26-33].

\section{Study characteristics}

Among children/adolescents, the first study was published in 2008 [34] and the most recent study was 2019 [30] (Table 1). The range of publication dates was wider among adults (1998-2019) (Table 2). All eight studies conducted among children/adolescents were conducted in San Francisco, CA, USA [26-30, 34-36], and all but one [26] were led by the same principal investigator (Chen) (Table 1). Among adults, one study was set in Australia [37], one in Canada [33], and one in South Korea [38], while all others were conducted in the United States [31, 32, 39-47] (Table 2). The average sample size was 60 and 63 among studies conducted in children/adolescents and adults, respectively (Tables 1 and 2). The average proportion of female participants was 50 and $64.5 \%$ among studies conducted in children/ adolescents and adults, respectively (Tables 1 and 2). The age range for interventions among children/adolescents was three to 18. Among children/adolescents, all interventions included both diet and physical activity components, while among adults, two interventions focused on diet exclusively while three interventions focused on physical activity exclusively (Tables 1 and 2). Among children/adolescents, intervention duration was 2 months for six studies and 6 months for two studies (Table 1). Among adults, intervention duration ranged from 5 weeks to 1 year, with most common duration of 6 months in four studies (Table 2).

\section{Risk of bias within studies}

Among studies conducted in children/adolescents (Fig. 2 and b), only Chen 2018 [30] had low risk of bias for all criteria. Four of the studies were not evaluated for random sequence generation, allocation concealment, or blinding, as they were not randomized controlled trials. Four studies had a high risk of bias for incomplete outcome data (attrition bias).

Among studies conducted in adults (Fig. 3a and b), all of the studies had at least one criterion with a high risk of bias. Six of the studies were not evaluated for random sequence generation, allocation concealment, or blinding, as they were not randomized controlled trials. Common criteria rated with a high risk of bias was blinding of outcome assessment (six studies), incomplete outcome data (ten studies), and selective reporting (five studies).

\section{Intervention characteristics}

Among children/adolescents, four studies were randomized controlled trials, three studies were pre-post singlearm interventions, and one study included a historical control group (Table 3). The most common intervention was iStart Smart, which was adapted for Chinese American children based on the National Institute of Health's WeCan! program (educational play-based activities teaching self-efficacy, critical thinking, and problem solving skills related to nutrition, physical activity, and coping) $[29,30,35,36]$. Intervention components included short video clips with hands-on activities to reinforce concepts; interactive dietary software (The Wok); and 60 min exercise classes (basketball, dodge ball, badminton) weekly for 8 sessions. Study participants were provided with a pedometer, activity diary, and books related to physical activity. A one-hour parent workshop was also included to provide reinforcement and social support. Theoretical models included the Ecological Model of Childhood Obesity, Social Cognitive Theory (five studies), Transtheoretical model, and InformationMotivation-Behavior Models (Table 3).

Among adults, three studies were randomized controlled trials, nine studies were pre-post single-arm interventions, and two studies were two-group repeated measures quasi-experimental design (Table 4). Interventions included adaptations of the Diabetes Prevention Program [32, 37, 39, 45] DASH diet [33], a cancer survival program [41], diabetes management programs [43, 46, 47], walking programs [38, 40], community-based 


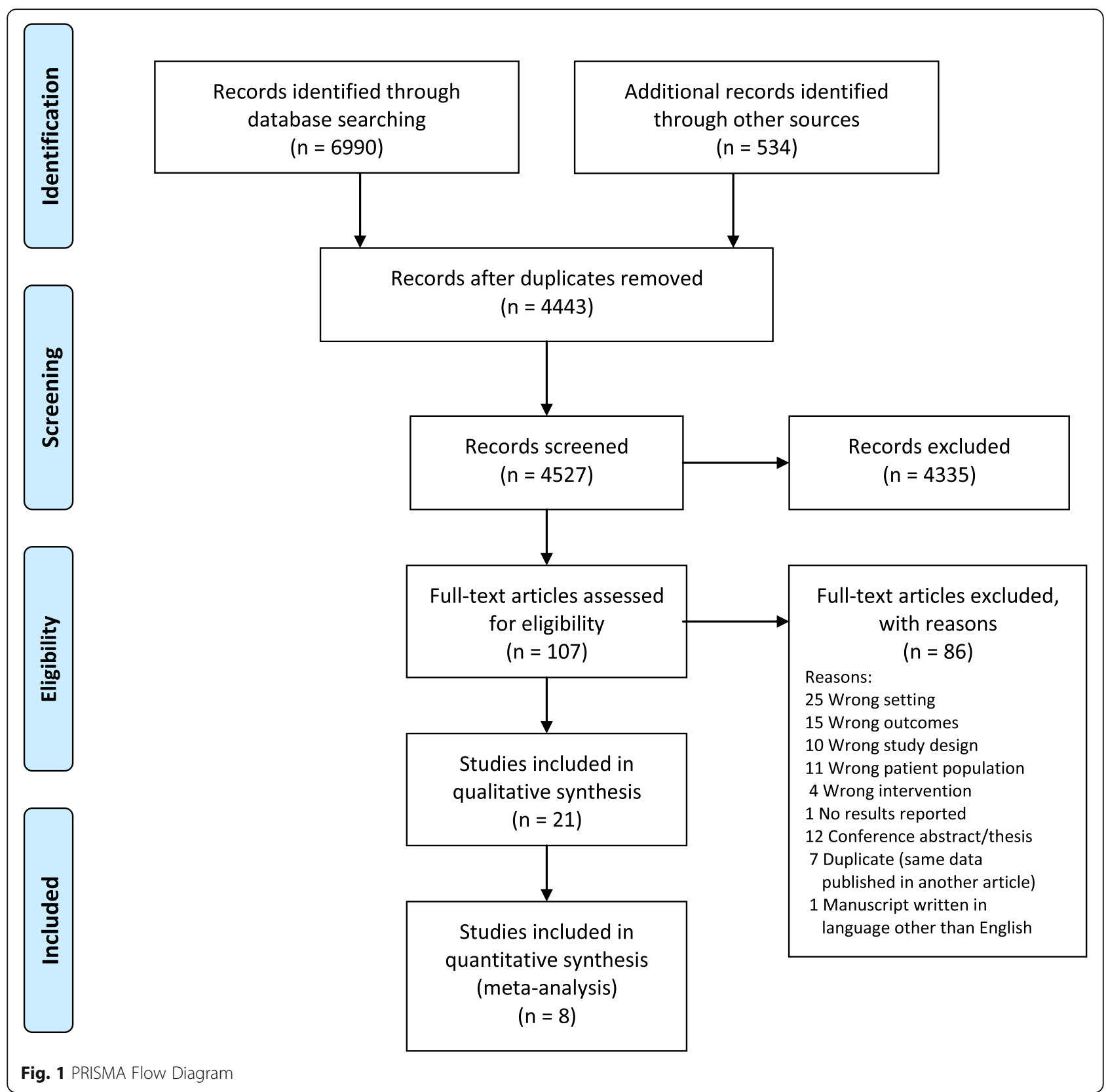

programs [42], tai chi [44], and an intervention to incorporate more brown rice in the diet [31]. Theoretical models included Transtheoretical Model, Culture Care Theory, Chronic Care model, Theory of reasoned action, Orem's theory of self care, Empowerment model, REAIM, Social Cognitive Theory, and traditional Chinese Medicine principles (Table 4).

\section{Intervention effectiveness}

Among children/adolescents, sufficient data were available for meta-analysis for BMI, WHR, SBP, and DBP. The pooled effect including five studies did not show significant changes in BMI (effect size $=-0.27 \mathrm{~kg} / \mathrm{m}^{2}$;
(95\%CI -0.91, 0.36) (Fig. 4a). For WHR, there were also no significant changes over time between groups, (two pooled studies with an effect size -0.01 (95\%CI -0.03, 0.00 ). There was also no significant effect of intervention in terms of changes in SBP or DBP as the pooled effect across three studies was $-3.41 \mathrm{mmHg}(95 \% \mathrm{CI}-9.40$, $2.58)$ and $-4.58 \mathrm{mmHg}(95 \% \mathrm{CI}-9.56,0.41)$, respectively. Results did not substantively change in sensitivity analyses using 0.5 and 0.8 as the correlation between baseline and follow-up measures (data not shown). For the other outcomes of interest (WC, LDL, HDL, and FBG) (Table 5), just one study reported findings, and statistically significant differences were only reported for HDL. 


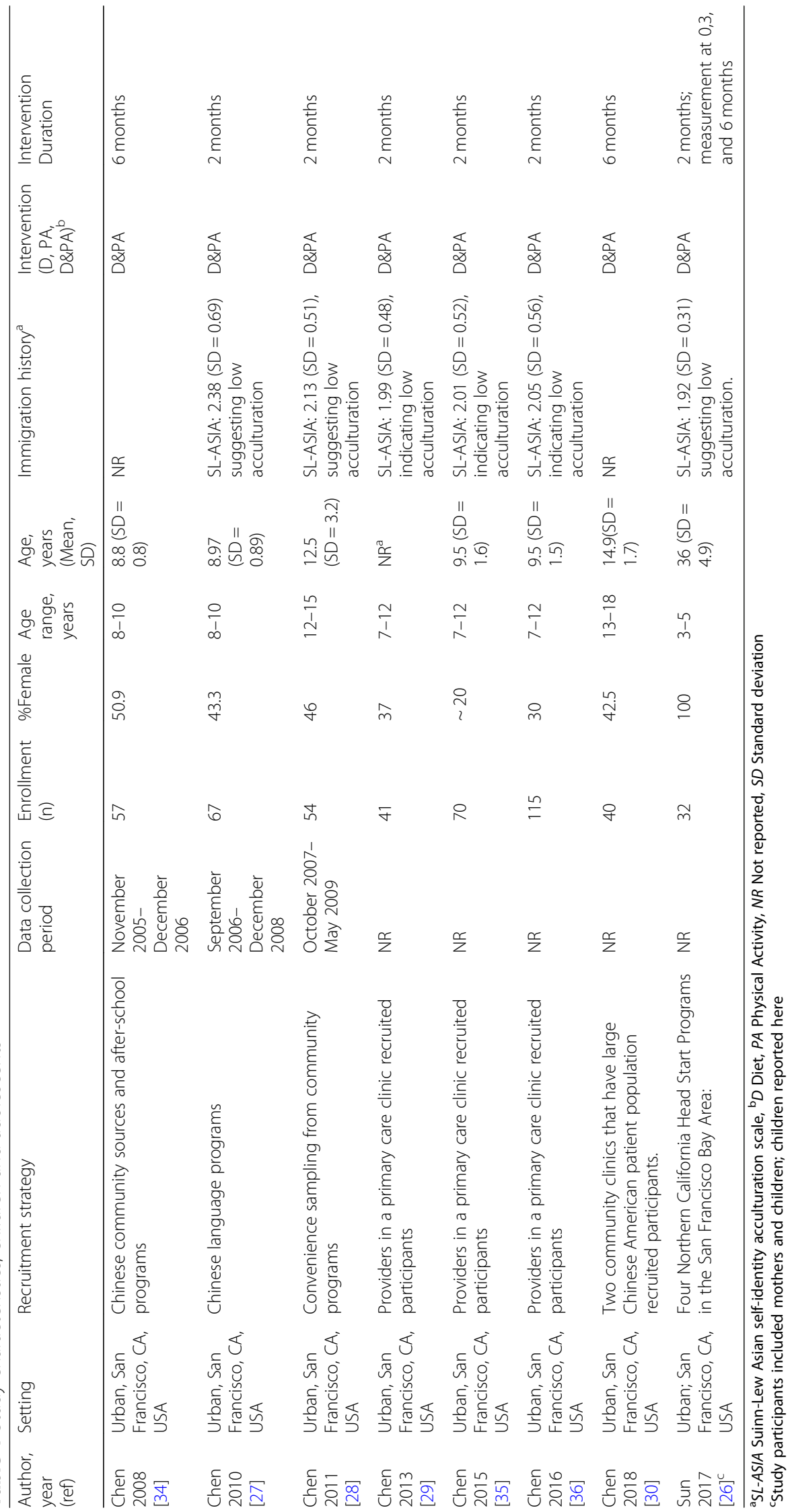




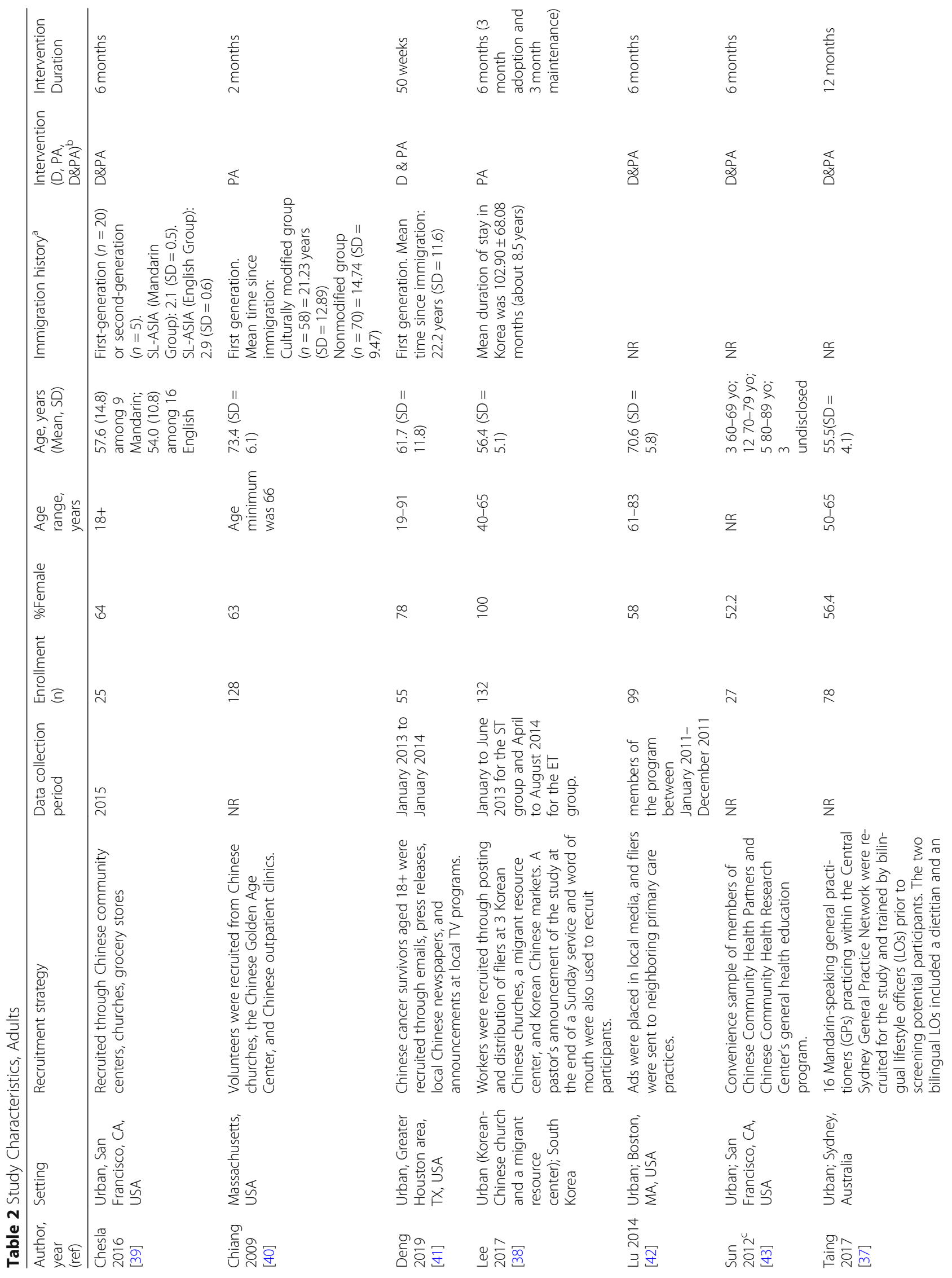




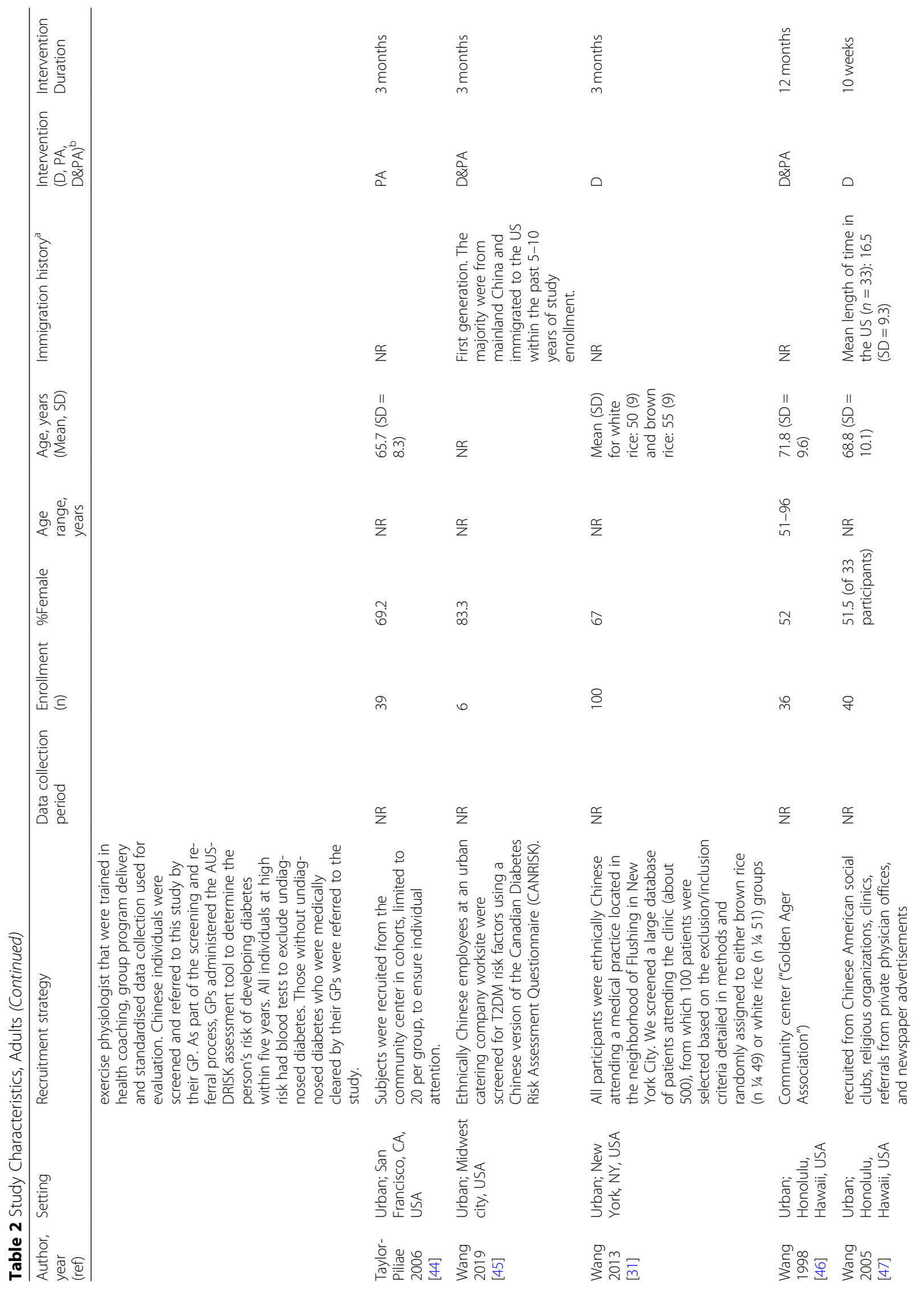




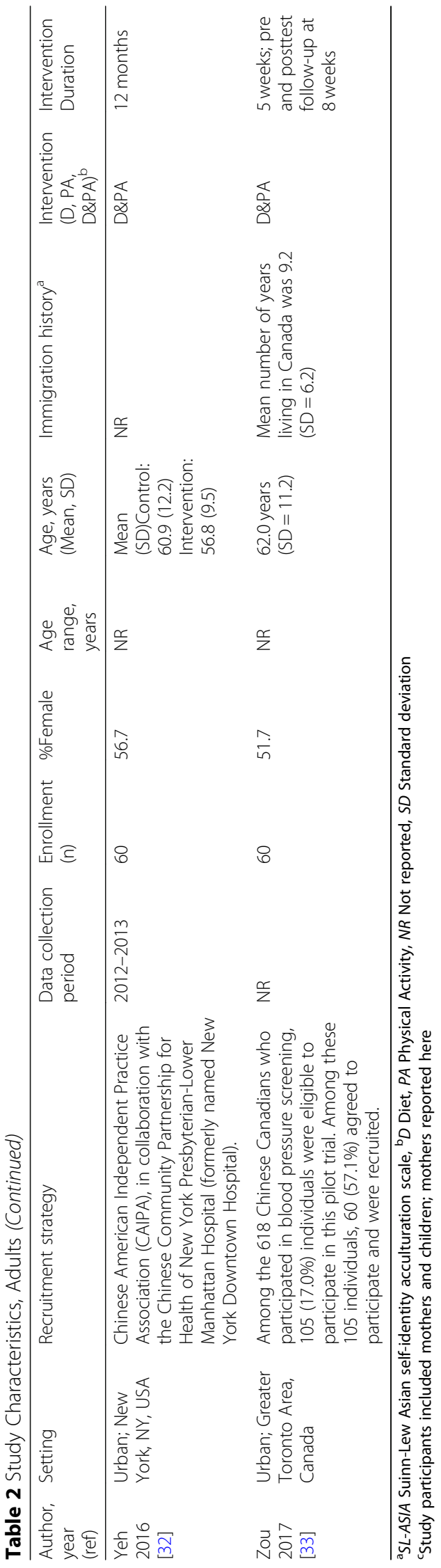



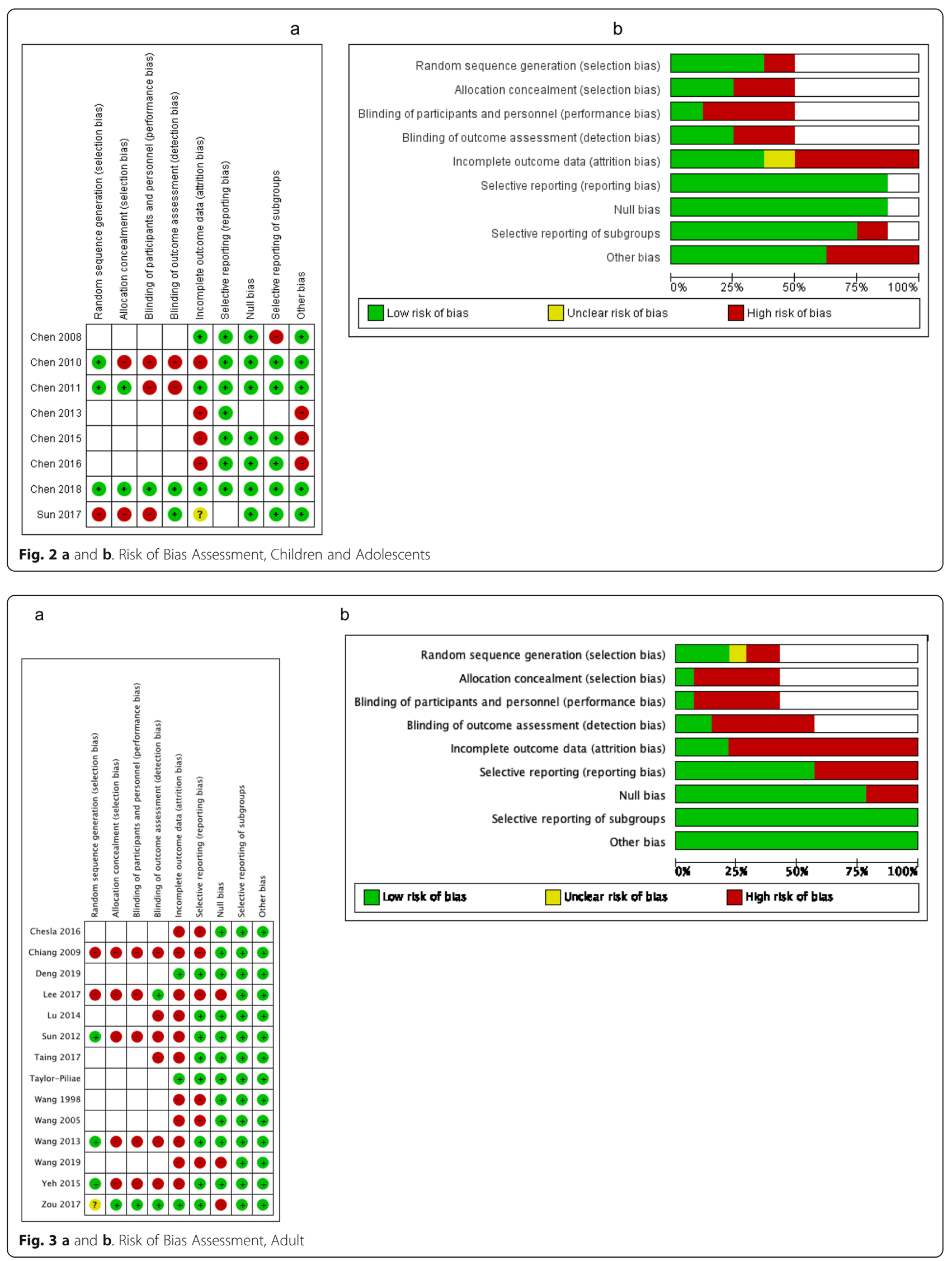


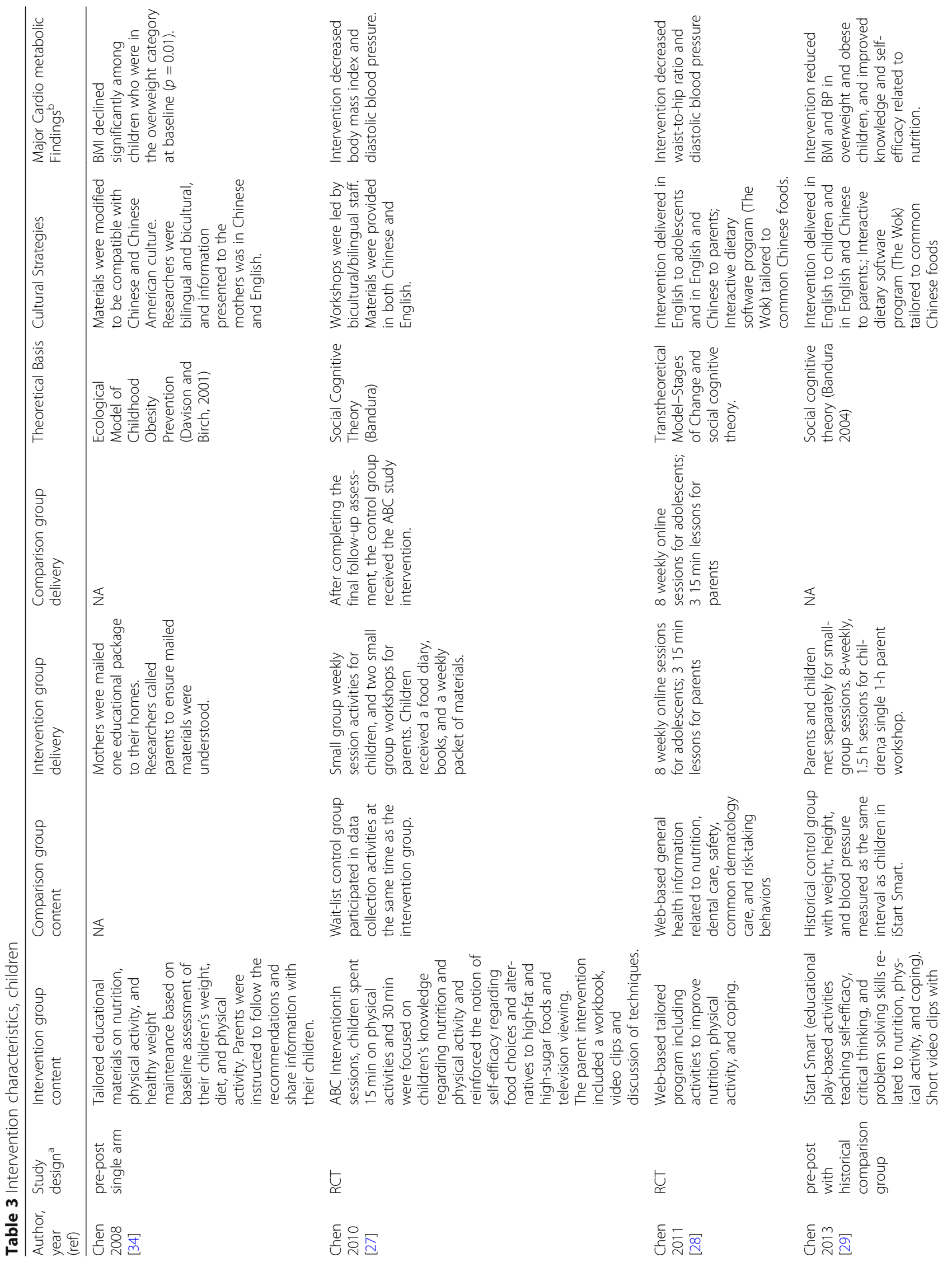




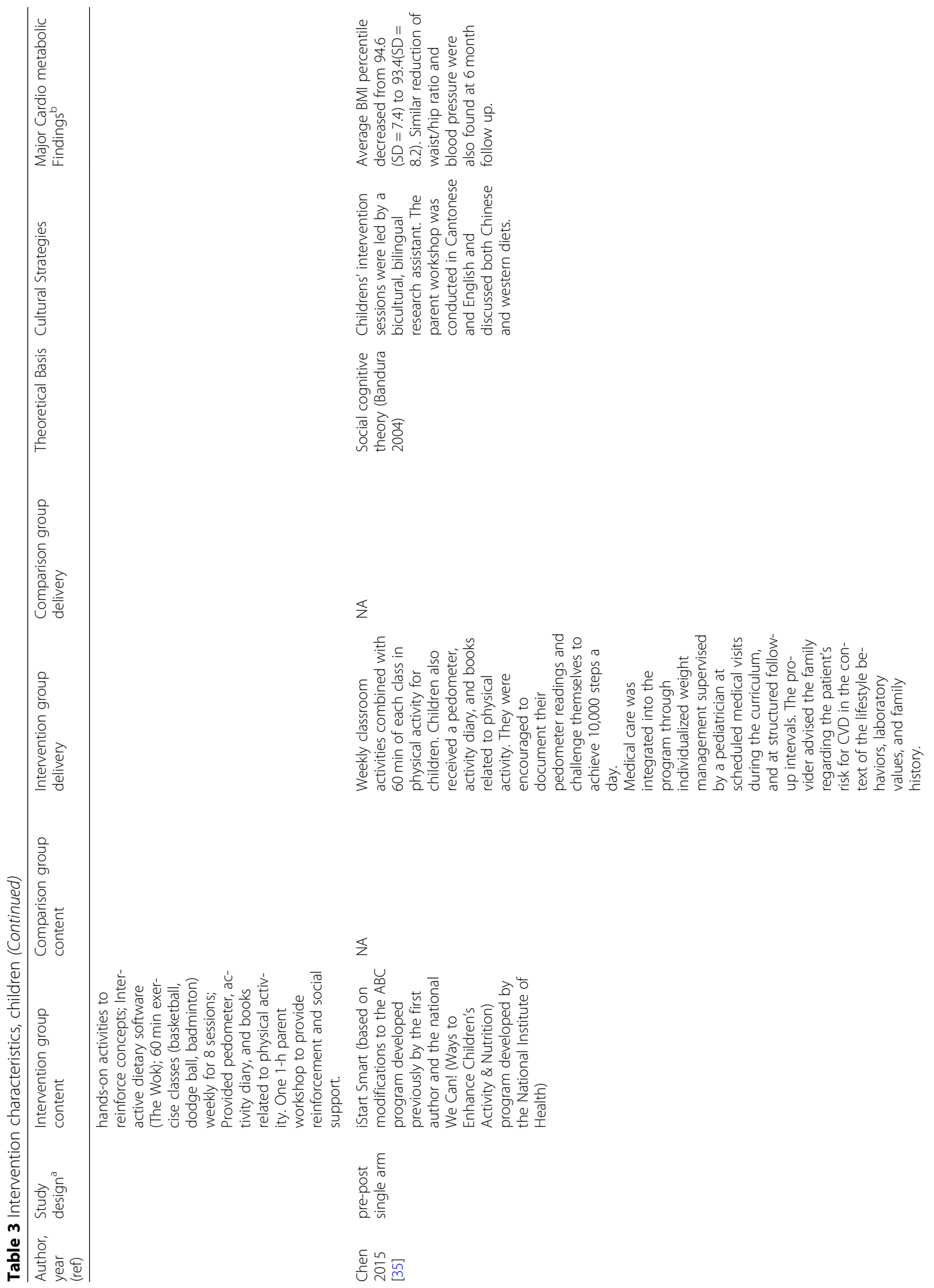




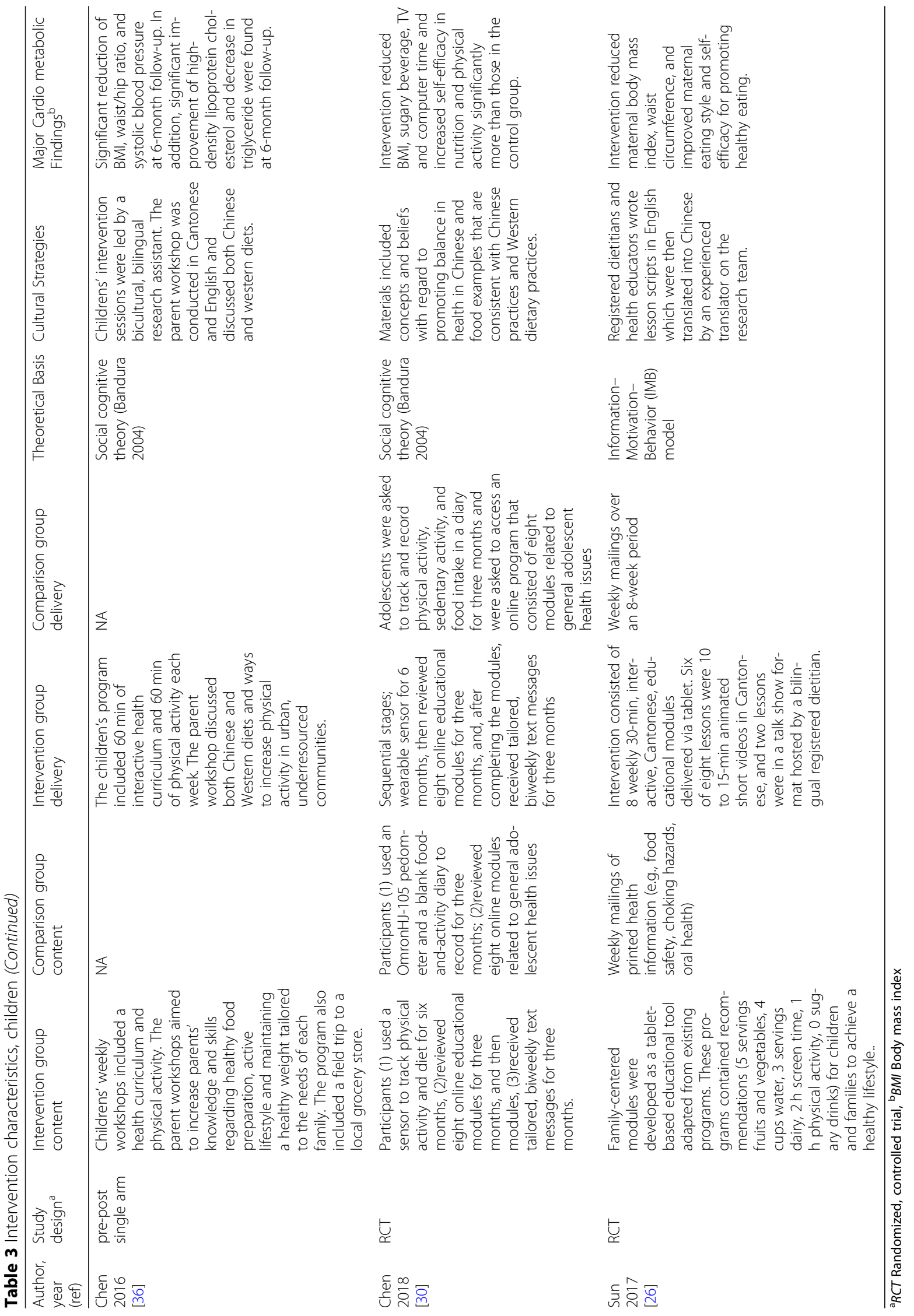




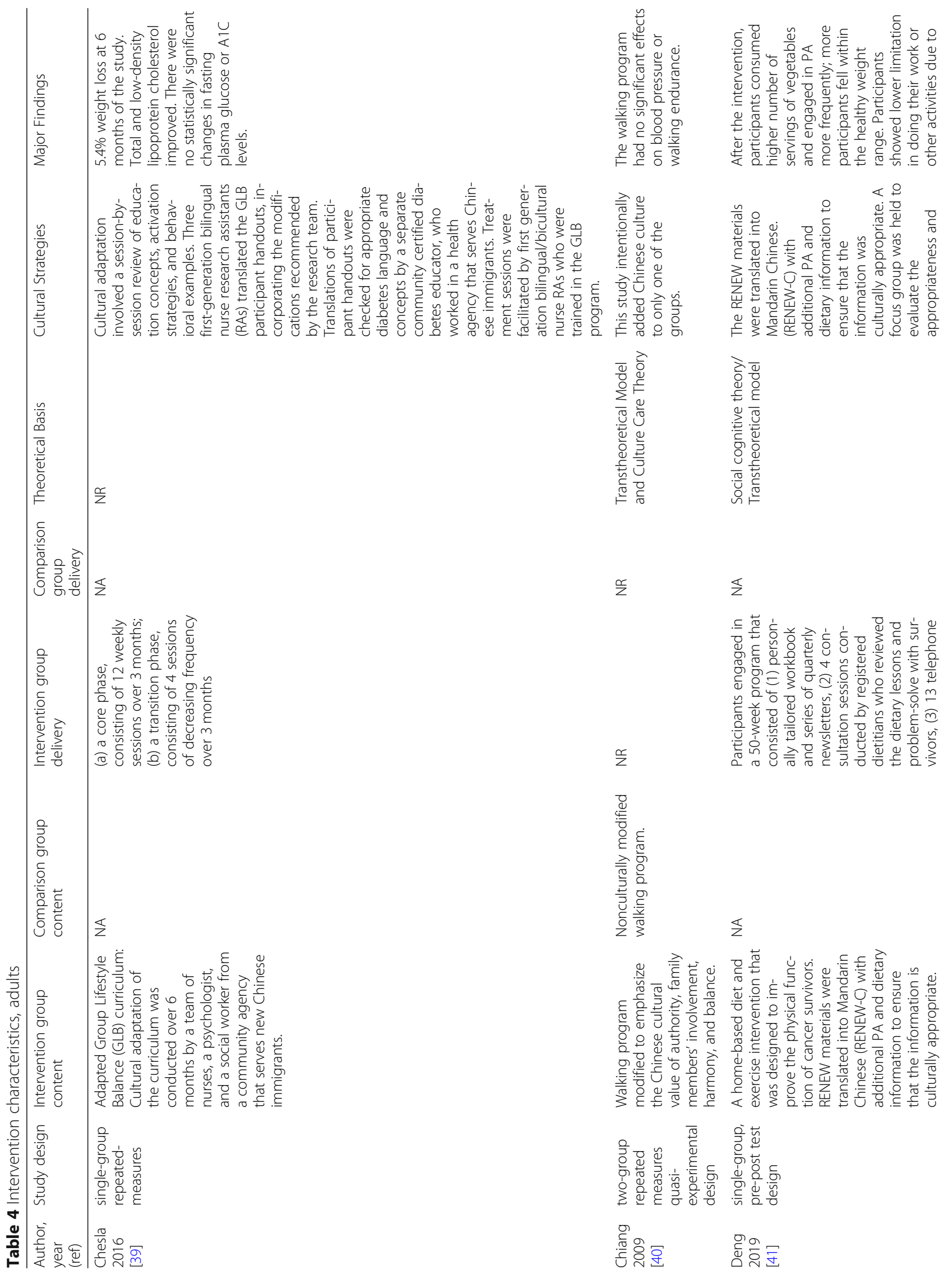




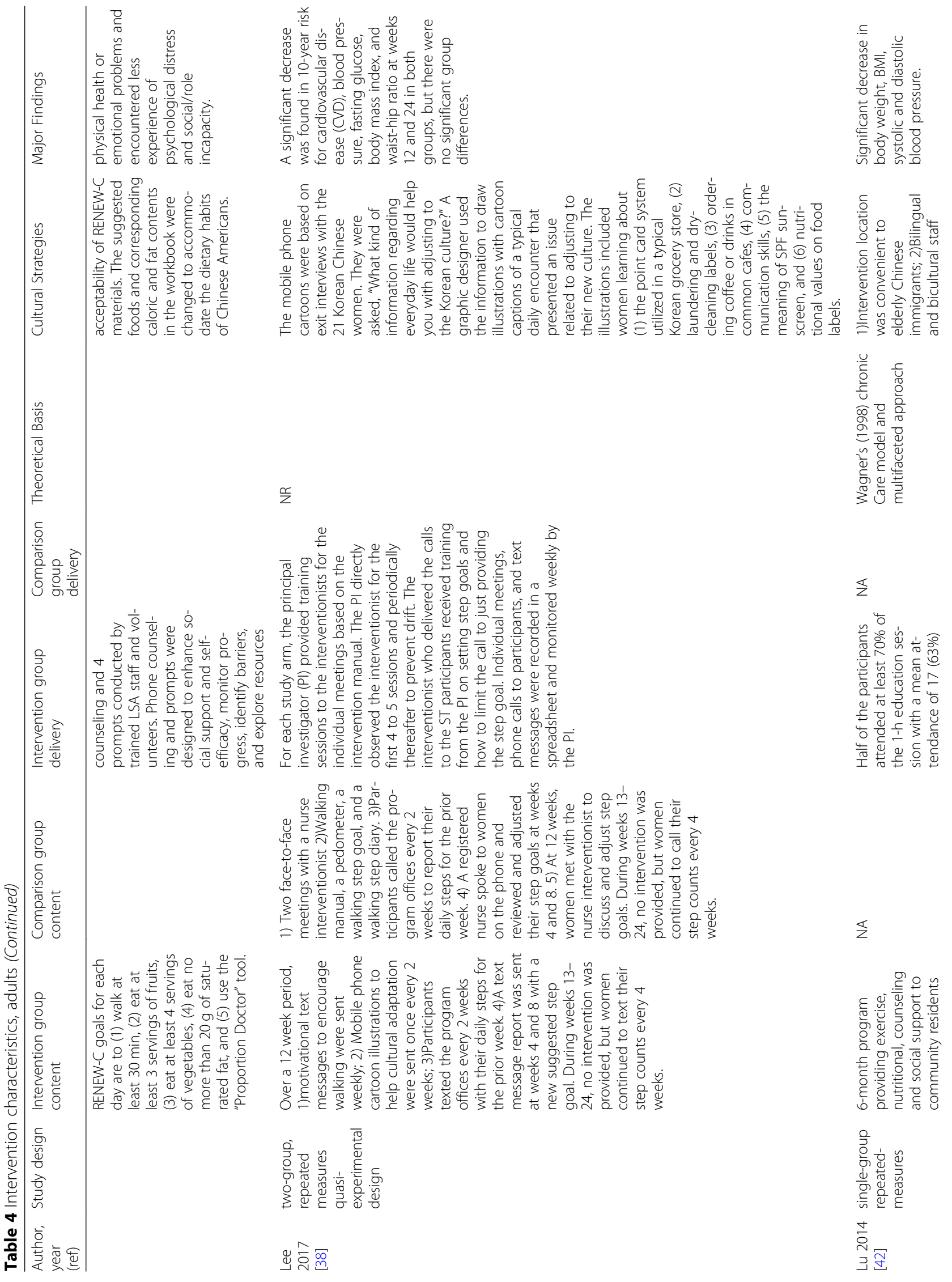




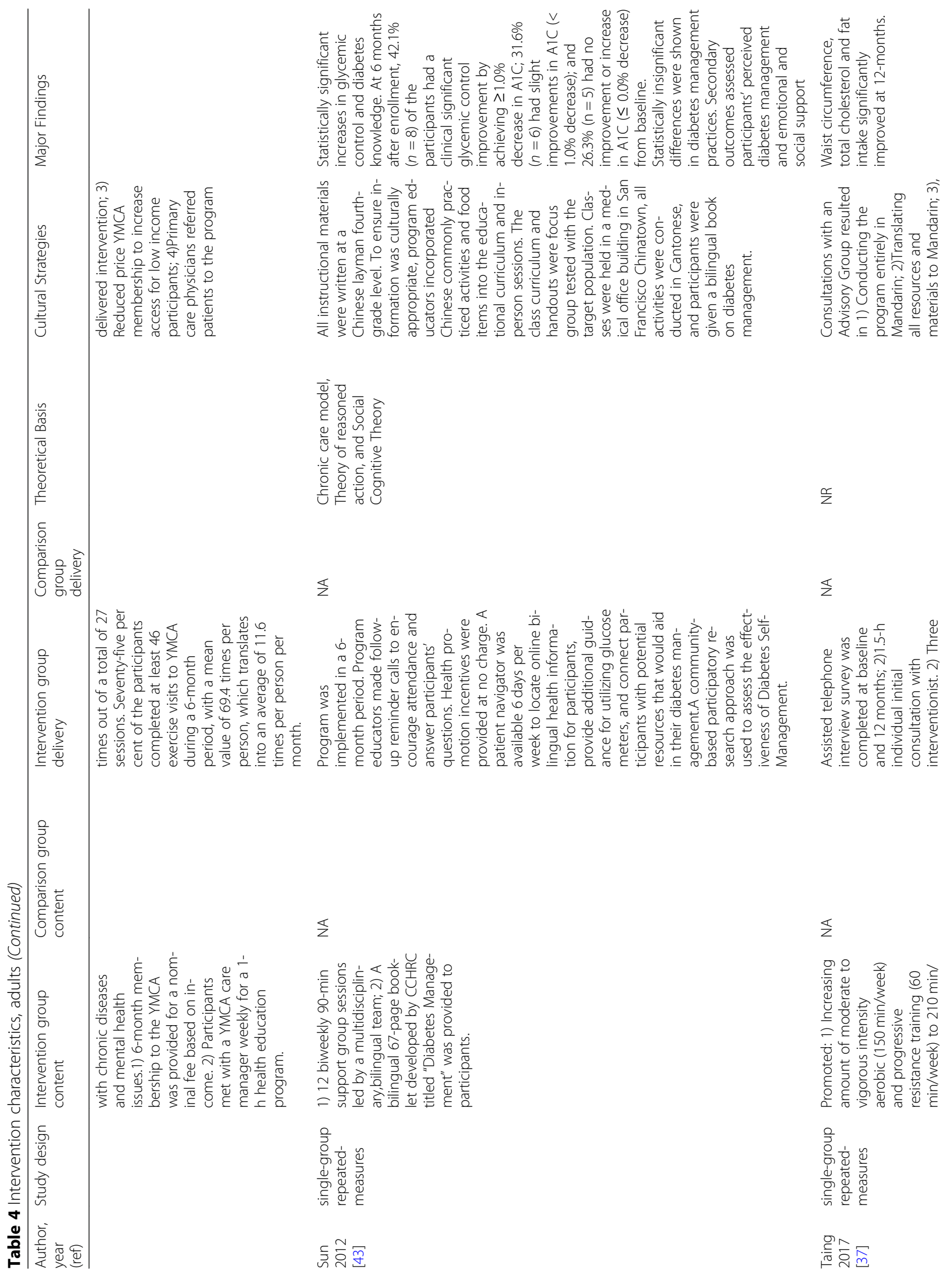




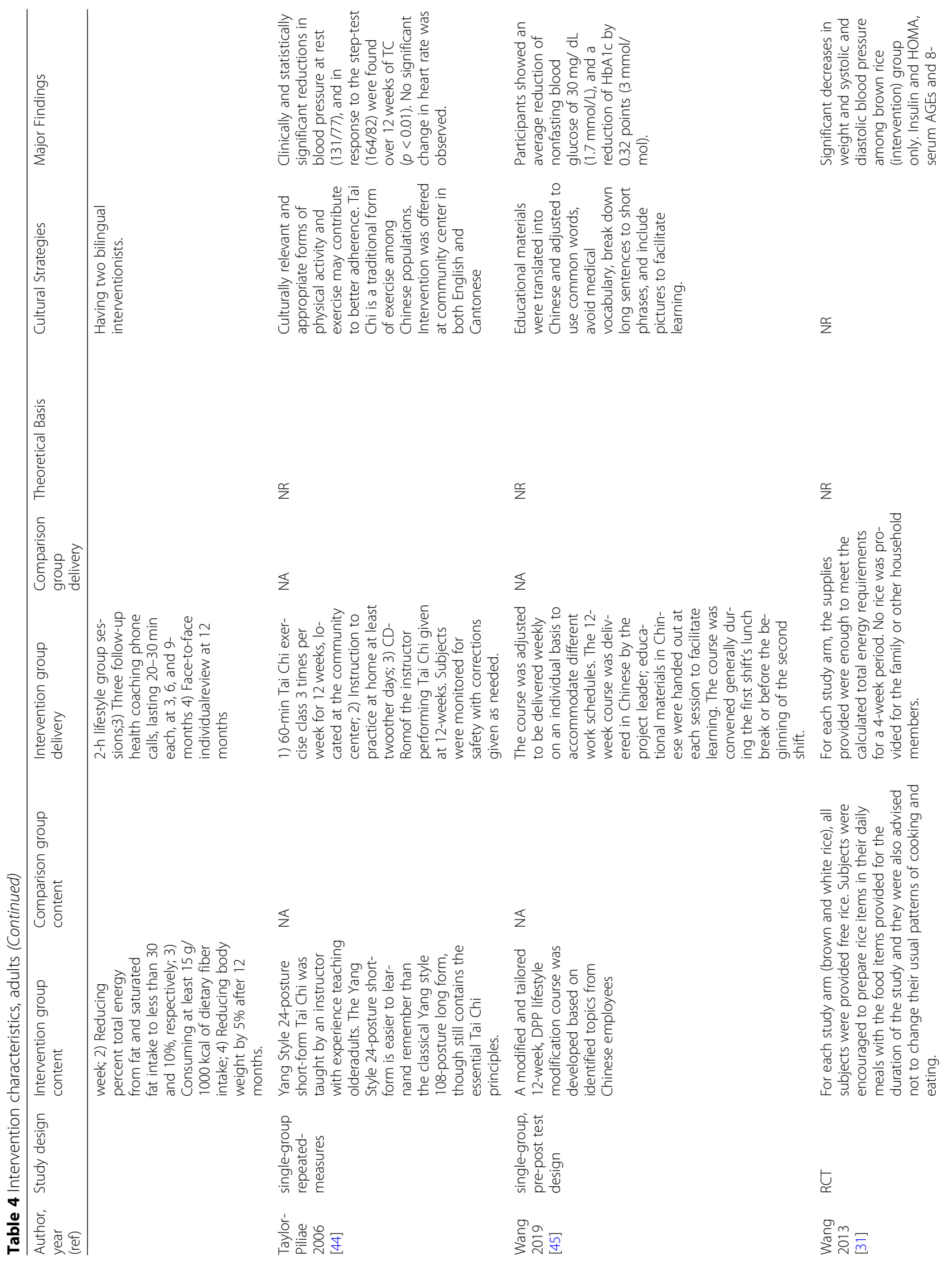




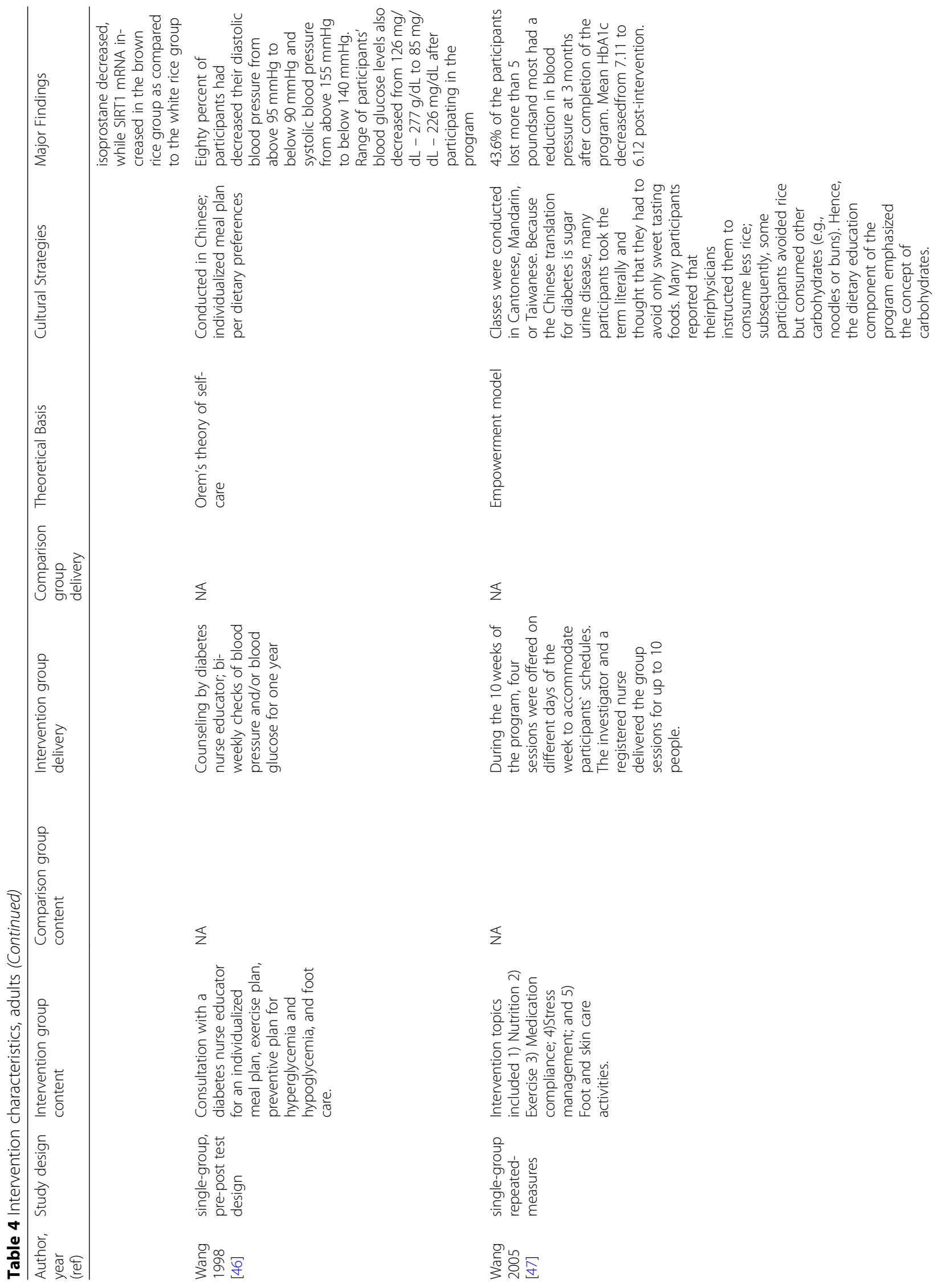




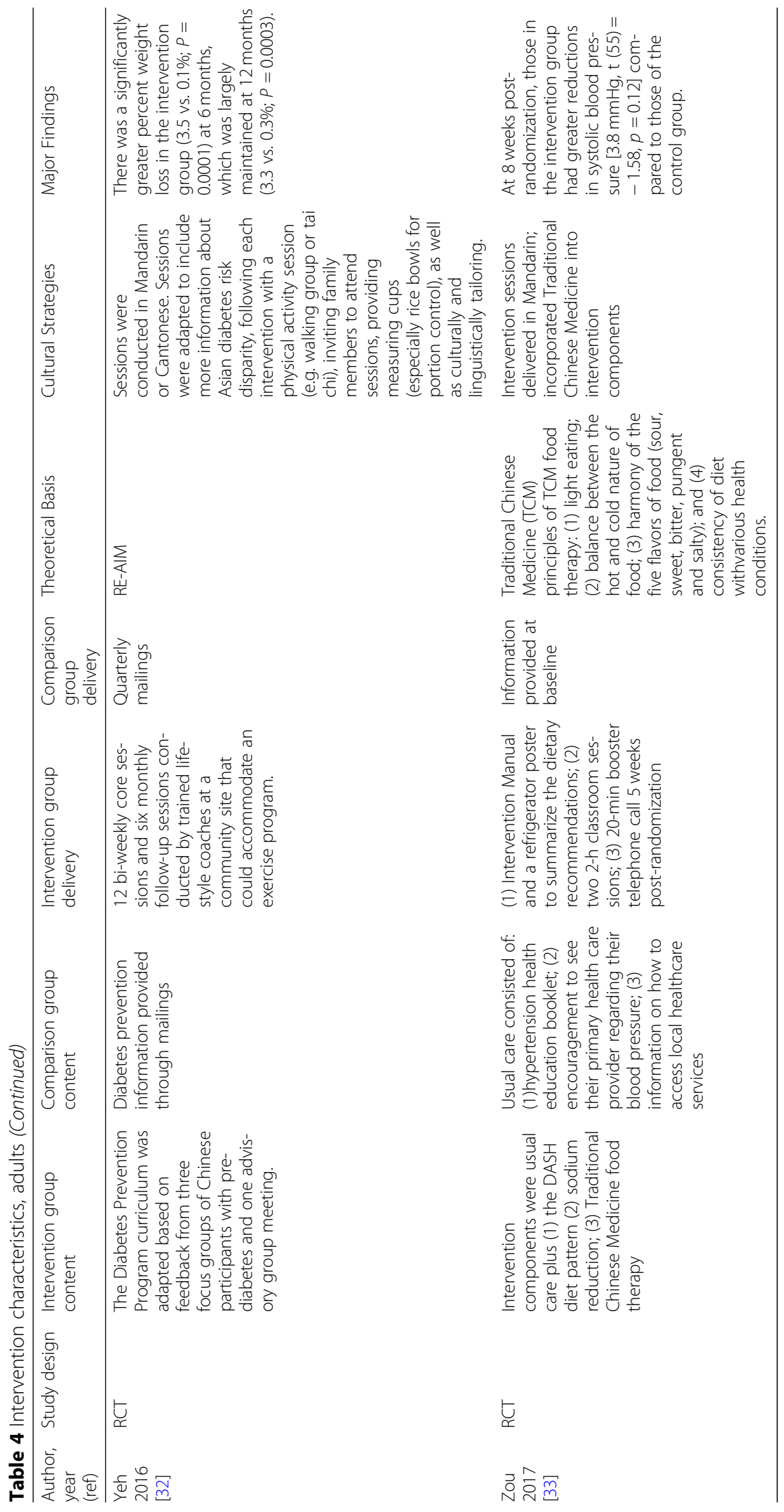




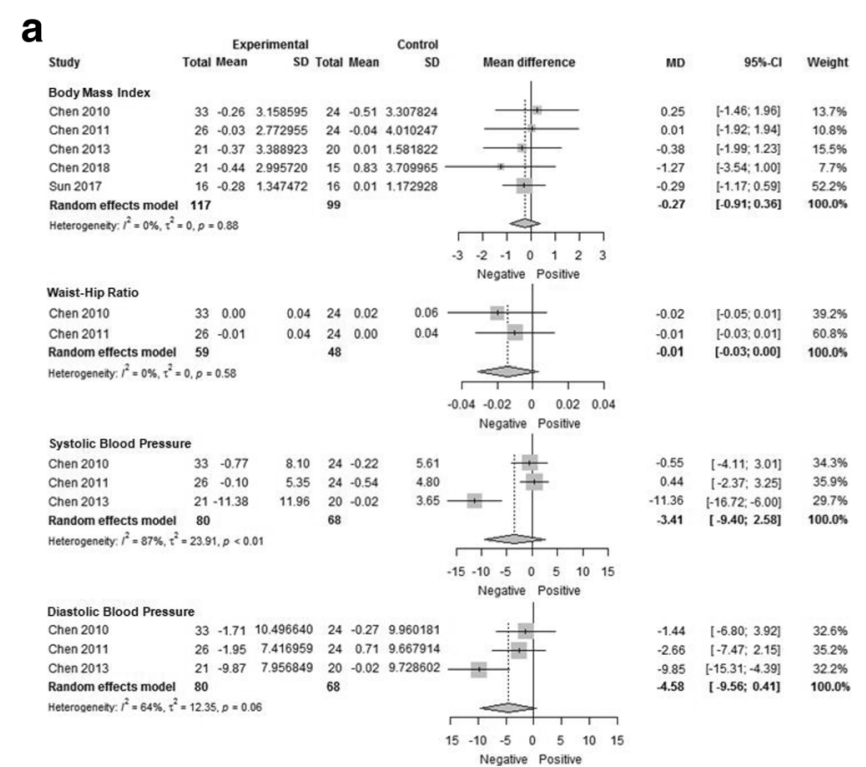

b

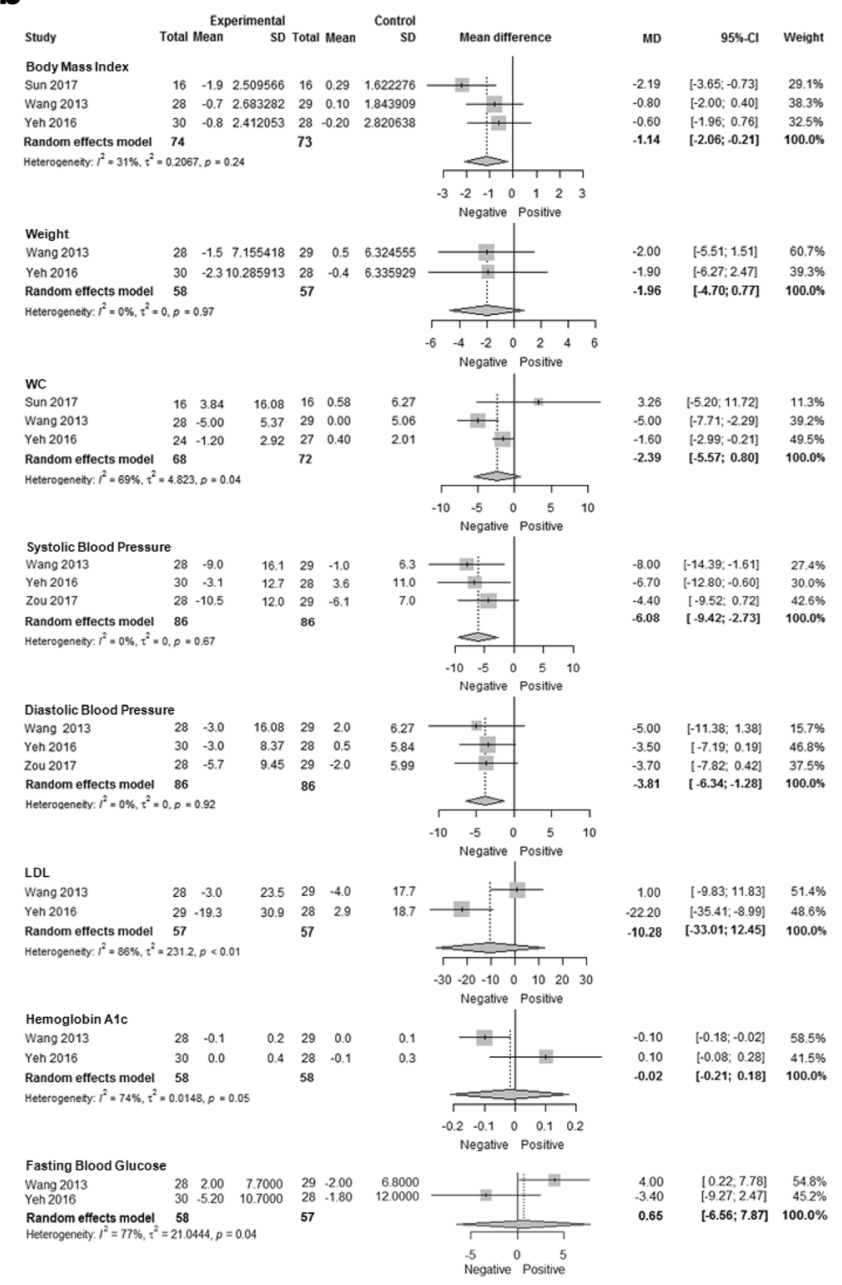

Fig. 4 a Meta-analysis of mean change in cardiometabolic outcomes from baseline to post-intervention for Chinese migrant children/ adolescents. b Meta-analysis of mean change in cardiometabolic outcomes from baseline to post-intervention for Chinese migrant adults 
Table 5 Cardiometabolic outcomes- children

\begin{tabular}{|c|c|c|c|c|c|c|c|c|c|c|c|c|c|}
\hline & \multirow[b]{3}{*}{ Author, year } & \multicolumn{6}{|c|}{ Intervention group } & \multicolumn{6}{|c|}{ Control group } \\
\hline & & \multicolumn{3}{|l|}{ Baseline } & \multicolumn{3}{|c|}{ Post-intervention } & \multicolumn{3}{|c|}{ Baseline } & \multicolumn{3}{|c|}{ Post-intervention } \\
\hline & & Mean & SD & $n$ & Mean & SD & $n$ & Mean & SD & $n$ & Mean & SD & $n$ \\
\hline \multirow[t]{7}{*}{$\mathrm{BMI}\left(\mathrm{kg} / \mathrm{m}^{2}\right)$} & Chen 2010 & 19.74 & 3.58 & 35 & 19.48 & 3.48 & 33 & 18.65 & 2.63 & 32 & 18.14 & 2.60 & 24 \\
\hline & Chen 2011 & 20.79 & 3.12 & 26 & 20.76 & 3.08 & 26 & 20.25 & 3.21 & 24 & 20.21 & 3.13 & 24 \\
\hline & Chen 2013 & 25.53 & 3.65 & 21 & 25.16 & 3.91 & 21 & 23.17 & 1.22 & 20 & 23.18 & 1.28 & 20 \\
\hline & Chen 2015 & 24.03 & 3.47 & 70 & 23.67 & 3.52 & 70 & & & & & & \\
\hline & Chen 2016 & 23.7 & 3.6 & 115 & 23.4 & 3.5 & 115 & & & & & & \\
\hline & Chen 2018 & 27.37 & 3.26 & 23 & 26.93 & 3.43 & 21 & 28.35 & 4.36 & 17 & 29.18 & 3.88 & 15 \\
\hline & Sun 2017 & 16.86 & 1.57 & 16 & 16.58 & 1.43 & 16 & 16.24 & 1.28 & 16 & 16.25 & 1.34 & 16 \\
\hline WC (cm) & Chen 2013 & 82.63 & 11.25 & 21 & 81.33 & 10.77 & 21 & & & & & & \\
\hline \multirow[t]{4}{*}{ WHR } & Chen 2010 & 0.88 & 0.04 & 35 & 0.88 & 0.04 & 33 & 0.89 & 0.06 & 32 & 0.91 & 0.06 & 24 \\
\hline & Chen 2011 & 0.91 & 0.04 & 26 & 0.90 & 0.04 & 26 & 0.89 & 0.04 & 24 & 0.89 & 0.04 & 24 \\
\hline & Chen 2015 & 0.92 & 0.06 & 70 & 0.91 & 0.06 & 70 & & & & & & \\
\hline & Chen 2016 & 0.95 & 0.09 & 115 & 0.94 & 0.09 & 115 & & & & & & \\
\hline LDL (mg/dL) & Chen 2016 & 101.92 & 34.23 & 115 & 100.69 & 36.29 & 115 & & & & & & \\
\hline $\mathrm{HDL}(\mathrm{mg} / \mathrm{dL})$ & Chen 2016 & 47.83 & 10.39 & 115 & 50.94 & 10.24 & 115 & & & & & & \\
\hline \multirow[t]{5}{*}{$\mathrm{SBP}(\mathrm{mmHg})$} & Chen 2010 & 105.74 & 9.01 & 35 & 104.97 & 9.10 & 33 & 99.87 & 5.81 & 32 & 99.65 & 6.63 & 24 \\
\hline & Chen 2011 & 102.02 & 5.9 & 26 & 101.92 & 6.05 & 26 & 101.13 & 4.55 & 24 & 100.59 & 5.86 & 24 \\
\hline & Chen 2013 & 106.9 & 5.75 & 21 & 95.52 & 14.49 & 21 & 101.33 & 4.56 & 20 & 99.64 & 2.80 & 20 \\
\hline & Chen 2015 & 104.5 & 8.8 & 70 & 98.3 & 11.8 & 70 & & & & & & \\
\hline & Chen 2016 & 104 & 8.8 & 115 & 99.8 & 10.9 & 115 & & & & & & \\
\hline \multirow[t]{5}{*}{$\mathrm{DBP}(\mathrm{mmHg})$} & Chen 2010 & 63.23 & 12.91 & 35 & 61.52 & 9.62 & 33 & 57.70 & 11.31 & 32 & 57.43 & 10.95 & 24 \\
\hline & Chen 2011 & 63.26 & 8.19 & 26 & 61.31 & 8.39 & 26 & 60.43 & 9.98 & 24 & 61.14 & 11.44 & 24 \\
\hline & Chen 2013 & 62.73 & 7.11 & 21 & 52.86 & 9.83 & 21 & 59.92 & 11.2 & 20 & 59.27 & 10.51 & 20 \\
\hline & Chen 2015 & 61.9 & 8.7 & 70 & 57.0 & 12.1 & 70 & & & & & & \\
\hline & Chen 2016 & 62.7 & 8.3 & 115 & 59.1 & 11.1 & 115 & & & & & & \\
\hline $\mathrm{FBG}(\mathrm{mg} / \mathrm{dL})$ & Chen 2016 & 85.89 & 5.24 & 115 & 85.52 & 6.21 & 115 & & & & & & \\
\hline
\end{tabular}

For the three single group design studies, Chen 2008 only reported changes in BMI stratified by overweight status [34], while the other two reported minor improvements in BMI and blood pressure (Table 5) [35, 36].

Among adults, sufficient data were available for metaanalysis for BMI, weight, WC, SBP, DBP, LDL, HgBA1c, and FBG. The pooled effect including three studies showed significant changes in BMI (effect size $=-1.14 \mathrm{~kg}$ / $\mathrm{m}^{2} ; 95 \% \mathrm{CI}-2.06,-0.21$ ) (Fig. 4b). In contrast, among the two studies reporting weight, the effect was null (effect size $=-1.96 \mathrm{~kg} ; 95 \% \mathrm{CI}-4.70,0.77)$. For waist circumference, there were also no significant changes over time between groups (three pooled studies with an effect size $2.39(95 \% \mathrm{CI}-5.57,0.80))$. There were significant effects of intervention in terms of changes in SBP and DBP, as the pooled effect across three studies was $-6.08 \mathrm{mmHg}$ $(95 \% \mathrm{CI}-9.42,-2.73)$ and $-3.81 \mathrm{mmHg}(95 \% \mathrm{CI}-6.34$, -1.28), respectively. Finally, there was no significant effect of intervention on LDL (effect size $=-10.28 \mathrm{mg} / \mathrm{dL}$;
95\%CI -33.01, 12.45), HgBA1c (effect size $=-0.02 \%$; $95 \%$ CI $-0.21,0.18$ ), or FBG (effect size $=0.65 \mathrm{mg} / \mathrm{dL}$; $95 \% \mathrm{CI}-6.56,7.87)$. Results did not substantively change in sensitivity analyses using 0.5 and 0.8 as the correlation between baseline and follow-up measures (data not shown).

For the eleven studies that were not randomized controlled trials (Table 6), minor improvements were documented in BMI, weight, LDL, SBP, DBP, FBG, and HgbA1c. However, without a rigorous comparison group, the effects cannot be attributed to the interventions delivered with certainty. Data from one of the studies was not included in Table 6 due to incompatibility of the scales used to measure outcomes [45].

\section{Discussion}

As of February 2020, there were 21 published studies describing behavioral diet and physical interventions in Chinese migrants living in high-income countries. The majority were conducted in adults $(n=13)$, and just 
Table 6 Cardiometabolic outcomes- Adults

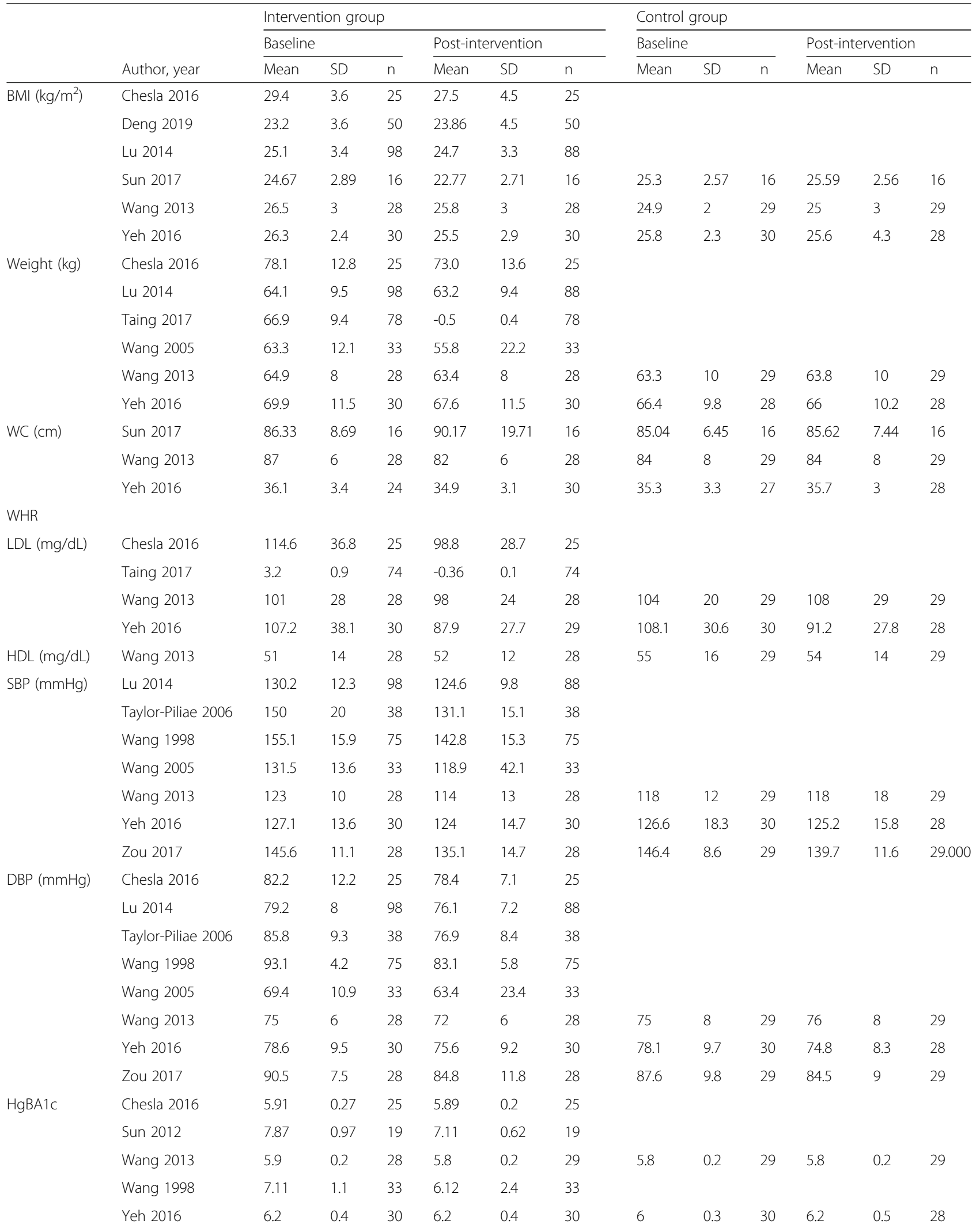


Table 6 Cardiometabolic outcomes- Adults (Continued)

\begin{tabular}{|c|c|c|c|c|c|c|c|c|c|c|c|c|c|}
\hline & \multirow[b]{3}{*}{ Author, year } & \multicolumn{6}{|c|}{ Intervention group } & \multicolumn{6}{|c|}{ Control group } \\
\hline & & \multicolumn{3}{|c|}{ Baseline } & \multicolumn{3}{|c|}{ Post-intervention } & \multicolumn{3}{|c|}{ Baseline } & \multicolumn{3}{|c|}{ Post-intervention } \\
\hline & & Mean & SD & $\bar{n}$ & Mean & SD & $n$ & Mean & SD & $\mathrm{n}$ & Mean & SD & $\mathrm{n}$ \\
\hline \multirow[t]{3}{*}{ FBG $(\mathrm{mg} / \mathrm{dL})$} & Chesla 2016 & 96.4 & 6.7 & 25 & 93.1 & 5.6 & 25.000 & & & & & & \\
\hline & Wang 2013 & 91 & 8 & 28 & 93 & 9 & 28 & 91 & 8 & 29 & 89 & 7 & 29 \\
\hline & Yeh 2016 & 109.7 & 8.8 & 30 & 104.5 & 13.3 & 30 & 103.3 & 11.7 & 30 & 101.5 & 14.5 & 28 \\
\hline HOMA-IR & Wang 2013 & 1.5 & 1.2 & 28 & 1.3 & 1.2 & 28 & 1.1 & 1 & 29 & 1.1 & 0.8 & 29 \\
\hline
\end{tabular}

*Results from Taing 2019 omitted, as post-intervention means and standard deviations weren't provided by the authors. Yeh 2016 results were obtained from the lead author

three of the adult interventions were conducted outside the United States (Australia, Canada, South Korea). Eight were conducted in children/adolescents; of these, seven were conducted by the same research group in San Francisco.

There were clinically meaningful changes in BMI [48] and blood pressure [49] among adults, but evidence was weak for other cardiometabolic outcomes (weight, WC, LDL, HgbA1c, and fasting glucose), and among children, there was no evidence of effect for any cardiometabolic outcomes. The intervention having the largest change in BMI among adults $(-2.19)$ had a much smaller effect on the offspring $(-0.29)$ [26]. Several explanations may help explain the differences in effects observed between adults and children in this study and others. First, postintervention measures were collected 3 months later in children, while mothers' BMI was collected immediately following the intervention. Second, BMI z-scores, which better account for growth stage compared to BMI among children, were not reported by the authors. Furthermore, most of the adult intervention periods were longer-term (6-12 months) whereas most of the studies conducted among children were 2 months in duration.

This report fills a gap in our understanding of the evidence base for behavioral diet and physical activity interventions conducted in Chinese migrants and their descendants living in high-income countries. Other reviews have examined diet and physical activity behaviors among African [50] and South Asian [51] migrants to high-income countries. For example, a review of the effects of diet and physical activity interventions on weight, BMI, and waist circumference among South Asian migrants including 29 studies also observed no significant differences among children but a significant improvement in weight only among adults (mean difference $-1.8 \mathrm{~kg}, 95 \% \mathrm{CI}-2.5$ to $-1.2 \mathrm{~kg}$ ) [51].

Limitations must be acknowledged in interpreting these findings. Despite searching seven databases and reference lists for all identified articles, it is possible that relevant studies were missed, if for example, the title or abstract didn't describe analyses specific to Chinese migrants. Although the characteristics of each intervention as are described in this review in order to help identify which intervention components might be effective, given the small sample size and heterogeneity of the studies, the review cannot definitively summarize successful strategies for behavioral diet and physical activity interventions targeted at Chinese-origin groups [52-55].

Most studies conducted a complete case analysis rather than accounting for loss to follow-up incorporating missing data methods such as multiple imputation. Complete case analyses would overestimate any effect of the intervention if, for example, participants who dropped out lost less weight compared to those who completed the study. We did not make any adjustment for how studies accounted for attrition in our analysis, but attrition bias was accounted for in the quality assessment. In summary, a major limitation of our analyses was having a relatively small number of controlled trials that were suitable for meta-analyses. We only included controlled trials, as opposed to single arm pre-post studies, in the meta-analyses to minimize the likelihood that observed changes in cardiometabolic outcomes were due to factors other than the intervention, particularly in growing children.

Suggestions for improvement include increased attention to (1) how interventions are culturally adapted; (2) the types of behavior change techniques and theories that are used to underpin interventions; (3) loss to follow-up by study arm; (4) variability within the Chinese-origin population, particularly with respect to generational differences that may be important for the design of interventions; and (5) contextual factors, such as whether the setting is rural or urban. These recommendations would enable reviewers to assess how behavior change techniques and theories moderate effectiveness, to assess the equity impacts of interventions, and to examine explanations for heterogeneity between interventions.

\section{Conclusions}

Given our mixed findings, more work is needed to support the design of successful interventions, particularly those targeting children and their families. The 
development of effective interventions may well require a great deal of qualitative and quantitative research on knowledge, attitudes, behaviors, and perceptions. More research is needed into the differential effects of lifestyle interventions for Chinese immigrants compared with other ethnicities.

\section{Supplementary information}

Supplementary information accompanies this paper at https://doi.org/10. 1186/s12889-020-08805-3.

Additional file 1: Supplemental Table 1 Ovid Medline Database Search Strategy

\begin{abstract}
Abbreviations
BMI: Body mass index; CA: California; CABI: Commonwealth Agricultural Bureaux International; Cl: Confidence Interval; D: Diet; DASH: Dietary Approaches to Stop Hypertension; DBP: Diastolic blood pressure; FBG: Fasting blood glucose; HDL: High density lipoprotein; HgbA1c: Hemoglobin A1c; HOMA-IR: Homeostatic Model Assessment of Insulin Resistance; kg: Kilogram; LDL: Low density lipoprotein; m: Meter; mg/ $\mathrm{dL}$ : Milligram per deciliter; mmHg: Millimeters of mercury; MD: Mean difference; NR: Not reported; NYC: New York City; PA: Physical activity PRISMA: Preferred Reporting Items for Systematic Reviews and MetaAnalyses; PROSPERO: PROSPective Register Of systematic reviews; RCT: Randomized, controlled trial; RE-AIM: Reach, Effectiveness, Adoption, Implementation, Maintenance; SBP: Systolic blood pressure; SD: Standard deviation; SL-ASIA: Suinn-Lew Asian self-identity acculturation scale; UK: United Kingdom; USA: United States of America; WC: Waist circumference; WHR: Waist hip ratio
\end{abstract}

\section{Acknowledgements}

The authors would like to thank Agnes Park and Muhammad El Shatanofy for assistance with tables and figures.

\section{Authors' contributions}

JMB, NA, TP, and JW conceived the study design and developed the PROSPERO protocol. TR developed the search strategy and conducted the search on all databases. JMB, JW, NA, and TP reviewed abstracts. JMB and JW extracted study details and outcome information. TP resolved discrepancies in quality assessment, and NA resolved discrepancies in outcomes extraction. NA conducted the outcomes analysis, and JMB drafted the manuscript. The authors read and approved the final manuscript.

\section{Funding}

This work was supported by a Durham Senior Research Fellowship COFUNDed between Durham University and the European Union under grant agreement number 609412 . The role of the funding body in the design of the study and collection, analysis, and interpretation of data and in writing the manuscript should be declared.

\section{Availability of data and materials}

The datasets used and/or analysed during the current study are available in the published literature.

\section{Ethics approval and consent to participate}

Not applicable.

\section{Consent for publication}

Not applicable.

\section{Competing interests}

The authors declare that they have no competing interests.

\section{Author details}

'Department of Medicine, NYU Grossman School of Medicine, 462 First Avenue CD 673, New York, NY 10016, USA. ²Department of Anthropology, Durham University, South Road, Durham DH1 3LE, UK. ${ }^{3}$ NYU Health Sciences
Library, NYU Grossman School of Medicine, 550 First Avenue, New York, NY 10016, USA

Received: 21 October 2019 Accepted: 29 April 2020

Published online: 29 June 2020

References

1. Jin K, Ding D, Gullick J, Koo F, Neubeck L. A Chinese immigrant paradox? Low coronary heart disease incidence but higher short-term mortality in Western-dwelling Chinese immigrants: a systematic review and metaanalysis. J Am Heart Assoc. 2015:4(12):e002568. https://doi.org/10.1161/ JAHA.115.002568 Available from: https://www.ahajournals.org/doi/full/1 0.1161/JAHA.115.002568?url_ver=Z39.88-2003\&rfr_id=ori:rid:crossref.org\&rfr_ dat=cr_pub\%3dpubmed.

2. Zaninotto $P$, Mindell J, Hirani V. Prevalence of cardiovascular risk factors among ethnic groups: results from the Health Surveys for England. Atherosclerosis. 2007;195(1):e48-57.

3. Jin K, Neubeck L, Gullick J, Koo F, Ding D. Marked differences in cardiovascular risk profiles in middle-aged and older Chinese residents: Evidence from a large Australian cohort. Int J Cardiol. 2017;227:347-54.

4. Lauderdale DS, Rathouz PJ. Body mass index in a US national sample of Asian Americans: effects of nativity, years since immigration and socioeconomic status. Int J Obes Relat Metab Disord. 2000;24(9):1188-94.

5. Bates LM, Acevedo-Garcia D, Alegria M, Krieger N. Immigration and generational trends in body mass index and obesity in the United States: results of the National Latino and Asian American Survey, 2002-2003. Am J Public Health. 2008;98(1):70-7.

6. Albrecht SS, Diez Roux AV, Kandula NR, Osypuk TL, Ni H, Shrager S. Immigrant assimilation and BMI and waist size: a longitudinal examination among Hispanic and Chinese participants in the multi-ethnic study of atherosclerosis. Obesity (Silver Spring). 2013;21(8):1695-703.

7. Chiu M, Maclagan LC, Tu JV, Shah BR. Temporal trends in cardiovascular disease risk factors among white, South Asian, Chinese and black groups in Ontario, Canada, 2001 to 2012: a population-based study. BMJ Open. 2015; 5(8):e007232.

8. Rao G, Powell-Wiley TM, Ancheta I, Hairston K, Kirley K, Lear SA, et al. Identification of Obesity and Cardiovascular Risk in Ethnically and Racially Diverse Populations: A Scientific Statement From the American Heart Association. Circulation. 2015;132(5):457-72.

9. Dogra S, Meisner BA, Ardern Cl. Variation in mode of physical activity by ethnicity and time since immigration: a cross-sectional analysis. Int J Behav Nutr Phys Act. 2010;7:75.

10. Stamatakis E. Physical Activity. Health Survey for England 2004: The Health of Minority Ethnic Groups 2006:237-62.

11. Yi SS, Roberts C, Lightstone AS, Shih M, Trinh-Shevrin C. Disparities in meeting physical activity guidelines for Asian-Americans in two metropolitan areas in the United States. Ann Epidemiol. 2015;25(9):656-60 e2.

12. Leung $G$, Stanner $S$. Diets of minority ethnic groups in the UK: influence on chronic disease risk and implications for prevention. Nutr Bull. 2011;36: 161-98.

13. Parackal S, Stewart J, Ho E. Exploring reasons for ethnic disparities in dietand lifestyle-related chronic disease for Asian sub-groups in New Zealand: a scoping exercise. Ethn Health. 2017;22(4):333-47.

14. Taylor VM, Yasui Y, Tu SP, Neuhouser ML, Li L, Woodall E, et al. Heart disease prevention among Chinese immigrants. J Community Health. 2007;32(5): 299-310.

15. Rosenmoller DL, Gasevic D, Seidell J, Lear SA. Determinants of changes in dietary patterns among Chinese immigrants: a cross-sectional analysis. Int J Behav Nutr Phys Act. 2011:8:42

16. Zou P. Diet and Blood Pressure Control in Chinese Canadians: Cultural Considerations. J Immigr Minor Health. 2017:19(2):477-83.

17. Satia JA, Patterson RE, Taylor VM, Cheney CL, Shiu-Thornton S, Chitnarong K, et al. Use of Qualitative Methods to Study Diet, Acculturation, and Health in Chinese-American Women. J Am Diet Assoc. 2000;100(8):934-40.

18. Wang-Schweig M, Kviz FJ, Altfeld SJ, Miller AM, Miller BA. Building a conceptual framework to culturally adapt health promotion and prevention programs at the deep structural level. Health Promot Pract. 2014;15(4): 575-84.

19. Moher D, Liberati A, Tetzlaff J, Altman DG, Group P. Preferred reporting items for systematic reviews and meta-analyses: the PRISMA statement. J Clin Epidemiol. 2009;62(10):1006-12. 
20. Bank TW. World Bank Country and Lending Groups: Country Classification. 2018 [August 1 2019]. Available from: https://datahelpdesk.worldbank.org/ knowledgebase/articles/906519-world-bank-country-and-lending-groups.

21. Higgins J, Green S. Cochrane Handbook for Systematic Reviews of Interventions. Available from: https://training.cochrane.org/handbook.

22. Booth A, Papaioannou D, Sutton A. Systematic approaches to successful literature review. London: Sage Publications; 2012.

23. Higgins J, Green S. Cochrane Handbook for Systematic Reviews of Interventions Version 5.1.0 [updated March 2011]; 2011.

24. DerSimonian R, Laird N. Meta-analysis in clinical trials. Control Clin Trials. 1986;7(3):177-88.

25. DerSimonian R, Laird N. Meta-analysis in clinical trials revisited. Contemp Clin Trials. 2015;45(Pt A):139-45.

26. Sun A, Cheng J, Bui Q, Liang Y, Ng T, Chen JL. Home-Based and Technology-Centered Childhood Obesity Prevention for Chinese Mothers With Preschool-Aged Children. J Transcult Nurs. 2017;28(6):616-24.

27. Chen $J$ L, Weiss S, Heyman MB, Lustig RH. Efficacy of a child-centred and family-based program in promoting healthy weight and healthy behaviors in Chinese American children: a randomized controlled study. J Public Health. 2010;32(2):219-29.

28. Chen JL, Weiss S, Heyman MB, Cooper B, Lustig RH. The efficacy of the web-based childhood obesity prevention program in Chinese American adolescents (Web ABC study). J Adolesc Health. 2011;49(2):148-54.

29. Chen JL, Kwan M, Mac A, Chin NC, Liu K. iStart smart: a primary-care based and community partnered childhood obesity management program for Chinese-American children: feasibility study. J Immigr Minor Health. 2013; 15(6):1125-8.

30. Chen JL, Guedes CM, Lung AE. Smartphone-based healthy weight management intervention for Chinese American adolescents: Short-term efficacy and factors associated with decreased weight. J Adolesc Health. 2019;64(4):443-9.

31. Wang B, Medapalli R, Xu J, Cai W, Chen X, He JC, et al. Effects of a whole rice diet on metabolic parameters and inflammatory markers in prediabetes. e-SPEN J. 2013;8(1):e15-20.

32. Yeh MC, Heo M, Suchday S, Wong A, Poon E, Liu G, et al. Translation of the Diabetes Prevention Program for diabetes risk reduction in Chinese immigrants in New York City. Diabet Med. 2016;33(4):547-51.

33. Zou P, Dennis CL, Lee R, Parry M. Dietary Approach to Stop Hypertension with Sodium Reduction for Chinese Canadians (DASHNa-CC): A Pilot Randomized Controlled Trial. J Nutr Health Aging. 2017;21(10):1225-32.

34. Chen JL, Weiss S, Heyman MB, Vittinghoff E, Lustig R. Pilot study of an individually tailored educational program by mail to promote healthy weight in Chinese American children. J Spec Pediatr Nurs. 2008;13(3):212-22.

35. Chen JL, Kwan M, Liu K, Yu F. The Feasibility and Efficacy of Healthy Weight Management Program for Low Income Chinese American Overweight and Obese Children in a Primary Care Clinic. J Pediatr Nurs. 2015;30(6):821-8.

36. Chen $\lrcorner$, Kwan M. Short-Term Efficacy and Correlates of Change in Health Weight Management Program for Chinese American Children. Clin Pediatr (Phila). 2016;55(5):463-9.

37. Taing CY, Gibson AA, Colagiuri S, Vita P, Cardona-Morrell M, Bauman A, et al. Primary analysis of the Mandarin-speaking sub-study within the Sydney diabetes prevention program. Diabetes Res Clin Pract. 2017;132:118-26.

38. Lee H, Cho S, Wilbur J, Kim J, Park CG, Lee YM, et al. Effects of culturally adaptive walking intervention on cardiovascular disease risks for middleaged Korean-Chinese female migrant workers. Arch Environ Occup Health. 2017;72(6):317-27.

39. Chesla CA, Chun KM, Kwong Y, Gay CL, Chi HL, Gu Y, et al. Cultural Adaptation of the Group Lifestyle Balance Program for Chinese Americans. Diabetes Educ. 2016;42(6):686-96.

40. Chiang CY, Sun FK. The effects of a walking program on older Chinese American immigrants with hypertension: a pretest and posttest quasiexperimental design. Public Health Nurs. 2009;26(3):240-8.

41. Deng F, Chen D, Swartz MC, Sun H. A Pilot Study of a Culturally Tailored Lifestyle Intervention for Chinese American Cancer Survivors. Cancer Control. 2019;26(1):1073274819895489.

42. Lu Y, Dipierro M, Chen L, Chin R, Fava M, Yeung A. The evaluation of a culturally appropriate, community-based lifestyle intervention program for elderly Chinese immigrants with chronic diseases: a pilot study. J Public Health (Oxf). 2014;36(1):149-55.
43. Sun AC, Tsoh JY, Saw A, Chan JL, Cheng JW. Effectiveness of a culturally tailored diabetes self-management program for Chinese Americans. Diabetes Educ. 2012;38(5):685-94.

44. Taylor-Piliae RE, Haskell WL, Froelicher ES. Hemodynamic responses to a community-based Tai Chi exercise intervention in ethnic Chinese adults with cardiovascular disease risk factors. Eur J Cardiovasc Nurs. 2006;5(2):165-74.

45. Wang Y, Buchholz SW, Murphy M, Moss AM. A Diabetes Screening and Education Program for Chinese American Food Service Employees Delivered in Chinese. Workplace Health Saf. 2019;67(5):209-17.

46. Wang CY, Abbott LJ. Development of a community-based diabetes and hypertension preventive program. Public Health Nurs. 1998;15(6):406-14

47. Wang CY, Chan SM. Culturally tailored diabetes education program for Chinese Americans: a pilot study. Nurs Res. 2005;54(5):347-53.

48. Jensen MD, Ryan DH, Apovian CM, Ard JD, Comuzzie AG, Donato KA, et al. 2013 AHA/ACC/TOS guideline for the Management of Overweight and Obesity in adults: a report of the American College of Cardiology/American Heart Association task force on practice guidelines and the Obesity Society. Circulation. 2014;129(25):S102-38.

49. Verdecchia P, Gentile G, Angeli F, Mazzotta G, Mancia G, Reboldi G. Influence of blood pressure reduction on composite cardiovascular endpoints in clinical trials. J Hypertens. 2010;28(7):1356-65.

50. Ngongalah L, Rankin J, Rapley T, Odeniyi A, Akhter Z, Heslehurst N. Dietary and physical activity Behaviours in African migrant women living in high income countries: a systematic review and framework synthesis. Nutrients. 2018;10(8):E1017. https://doi.org/10.3390/nu10081017 Available from: https://www.mdpi.com/2072-6643/10/8/1017.

51. Brown T, Smith S, Bhopal R, Kasim A, Summerbell C. Diet and physical activity interventions to prevent or treat obesity in South Asian children and adults: a systematic review and meta-analysis. Int J Environ Res Public Health. 2015;12(1):566-94.

52. Ahmad F, Shik A, Vanza R, Cheung A, George U, Stewart DE. Popular health promotion strategies among Chinese and East Indian immigrant women. Women Health. 2004;40(1):21-40.

53. Arora A, Liu MN, Chan R, Schwarz E. 'English leaflets are not meant for me': a qualitative approach to explore oral health literacy in Chinese mothers in Southwestern Sydney, Australia. Community Dent Oral Epidemiol. 2012; 40(6):532-41.

54. Banna JC, Gilliland B, Keefe M, Zheng D. Cross-cultural comparison of perspectives on healthy eating among Chinese and American undergraduate students. BMC Public Health. 2016;16(1):1015.

55. Chang E, Choi S, Kwon I, Araiza D, Moore M, Trejo L, et al. Characterizing Beliefs about Stroke and Walking for Exercise among Seniors from Four Racial/Ethnic Minority Communities. J Cross Cult Gerontol. 2018;33(4):387-410.

\section{Publisher's Note}

Springer Nature remains neutral with regard to jurisdictional claims in published maps and institutional affiliations.

Ready to submit your research? Choose BMC and benefit from:

- fast, convenient online submission

- thorough peer review by experienced researchers in your field

- rapid publication on acceptance

- support for research data, including large and complex data types

- gold Open Access which fosters wider collaboration and increased citations

- maximum visibility for your research: over $100 \mathrm{M}$ website views per year

At BMC, research is always in progress.

Learn more biomedcentral.com/submissions 